\title{
Negatively refracting chiral metamaterials: a review
}

\author{
Tom G. Mackay, ${ }^{\text {a,b }}$ Akhlesh Lakhtakia ${ }^{\text {b }}$ \\ ${ }^{a}$ University of Edinburgh, School of Mathematics and Maxwell Institute for Mathematical \\ Sciences, Edinburgh EH9 3JZ, United Kingdom \\ T.Mackay@ed.ac.uk \\ ${ }^{\mathrm{b}}$ Pennsylvania State University, Department of Engineering Science and Mechanics, \\ Nanoengineered Metamaterials Group, University Park, Pennsylvania 16802-6812, USA \\ akhlesh@psu.edu
}

\begin{abstract}
Chirality extends the class of negatively refracting metamaterials by endowing a richer palette of electromagnetic properties. Chiral metamaterials can support negative refraction, which must be assessed in light of the closely related phenomenons of negative phase velocity and counterposition. Two categories of chiral metamaterials are being examined these days: (a) homogeneous and homogenizable chiral materials, as exemplified by isotropic chiral materials, Faraday chiral materials, and materials with simultaneous mirror-conjugated and racemic chirality characteristics; and (b) structurally chiral materials, as exemplified by helicoidal bianisotropic materials and ambichiral materials. The planewave response of a half-space occupied by a chiral metamaterial is complex, and important distinctions between negative refraction, negative phase velocity, and counterposition emerge. @ 2010 Society of Photo-Optical Instrumentation Engineers. [DOI: 10.1117/6.0000003]
\end{abstract}

Keywords: negative phase velocity; ambichirality; Faraday chiral material; mirror conjugation; racemic chirality; counterposition; helicoidal bianisotropic materials; negative refraction.

Paper SR090107 received Jul. 21, 2009; revised manuscript received Sep. 19, 2009; accepted for publication Sep. 19, 2009; published online Jan. 14, 2010.

\section{Introduction}

Probably the first engineered composite materials were bronze alloys made by smelting copper and tin together more than 5000 years ago. Research on mechanical properties of composite materials has a very rich history, the earliest parts of which are shrouded in the mists of time. Glass too was perhaps created in the Bronze Age, and colored glasses fabricated by mixing some particulate material in silica date back to more than two millenniums ago. Systematic research on optical properties of composite materials began two centuries back, with the publication of the Arago-Biot formula [1]. Since then, theoretical and experimental research continues unabated [2-5].

A composite material must have at least two constituent materials. Each constituent material is chemically inert with respect to the others in its immediate proximity within the composite material. This restriction excludes molecularly pure materials containing two or more types of atoms from being classified as composite materials.

Metamaterials are also composite materials, but with a difference. A metamaterial exhibits response characteristics that either (i) are not observed in or (ii) are enhanced relative to the individual responses of its constituent materials [6]. This characterization of metamaterials captures the notion originally proposed by Walser who envisaged them as being artificial materials fabricated by first downscaling macroscopic material architectures to the submicron and the nanometer length scales and then combining different downscaled architectures into macroscopic materials that would exceed their conventional counterparts in performance [7]. Possessing a cellular morphology, nanoengineered metamaterials may satisfy

$1946-3251 / 2010 / \$ 25.00$ (c) 2010 SPIE 

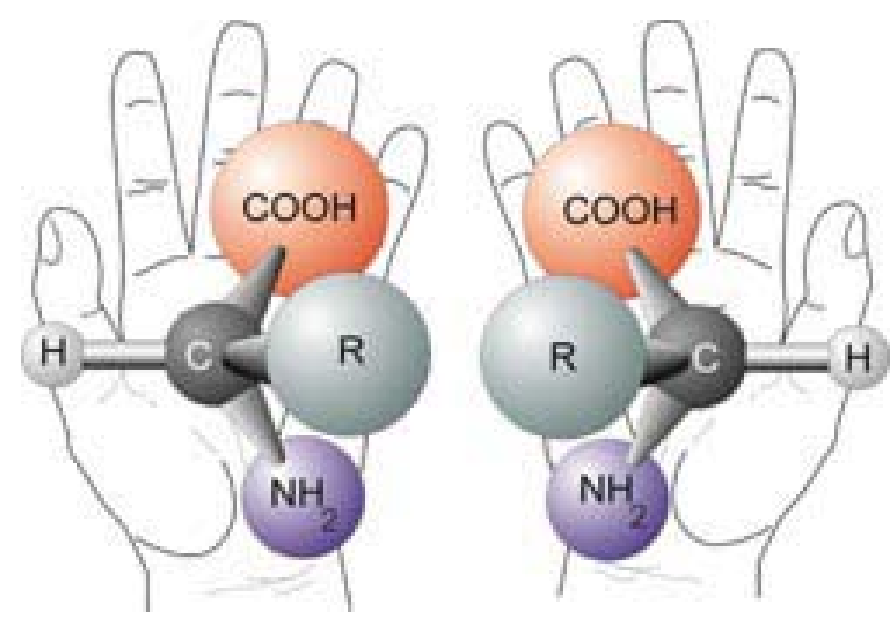

Fig. 1 Left-handed and right-handed enantiomers of a generic amino acid. (Courtesy of NASA.)

complex multifunctional requirements by a judicious juxtaposition of sufficiently small cells of different materials, shapes, and sizes [8].

Our focus in this review is on negatively refracting metamaterials. Millions of research dollars have been spent during the last decade on this topic, following experimental reports of negative refraction in certain metamaterials under microwave illumination $[9,10]$. Research activity has been greatly fuelled by the possibility of fabricating planar lenses with very high resolving power [11]. Within the past couple of years, metamaterials that exhibit negative refraction at optical frequencies have been developed [12-15], and current efforts are directed toward reducing the considerable dissipative losses associated with optical negative refraction [16,17]. Parenthetically, we note that negative refraction is not exclusive to artificial metamaterials: certain biological structures [18] and naturally occurring minerals [19] also exhibit negative refraction.

The simplest materials that exhibit negative refraction are isotropic dielectric-magnetic materials [20], but the scope for exotic behavior such as negative refraction is considerably increased if chirality is introduced [21-24], especially in combination with anisotropy $[25,26]$ or nonhomogeneity $[27,28]$. We confine ourselves in this review to chiral and certain related metamaterials - that too of the linear, passive kind.

An object is said to be chiral if it cannot be made to coincide with its mirror image by translations and/or rotations. A remarkable property of chiral materials is that they can discriminate between left-handed and right-handed electromagnetic waves [29]. In order to achieve this discrimination, handedness is necessarily intrinsic to chiral materials. Handedness is manifest in the microstructure of homogeneous and homogenizable chiral materials. For example, an isotropic chiral material comprises a random dispersion of handed molecules or inclusions [29]. An illustration of handed molecules is provided in Fig. 1. In contrast, handedness is manifest at the macroscopic level in structurally chiral materials. For example, the molecules of cholesteric liquid crystals are randomly positioned but macroscopically they exhibit a helicoidal orientational order [30,31], as represented in Fig. 2. Other examples of structurally chiral materials can be fabricated either as stacks of uniaxial laminas [32] or using thin-film technology [33]. Remarkably, artificial examples of both types of chiral materials were produced by Bose more than 11 decades ago [34].

Parenthetically, a third type of "chiral" medium has recently entered scientific literature $[35,36]$. Such a material is made by depositing spirals (and similar objects) on some flat surface. Spirals, being essentially two-dimensional objects, cannot be chiral, and "planar chirality" $[37,38]$ is an infelicitous term that ought to be replaced by a meaningful term. 


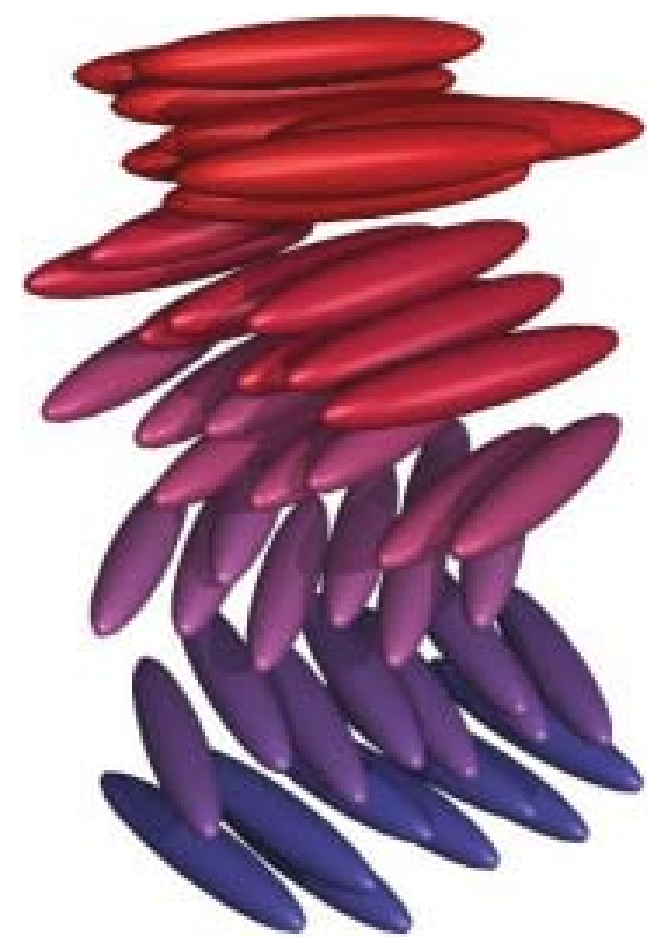

Fig. 2 A representation of a cholesteric liquid crystal (also known as a chiral nematic liquid crystal) that is structurally chiral. Adjacent liquid crystal molecules are twisted relative to their neighbors. (Courtesy of Kevin G. Yager, National Institute of Standards and Technology, Gaithersburg, MD, USA.)

In the following sections, we highlight the propensity of chiral metamaterials - of the homogeneous and homogenizable type and of the structurally chiral type- to exhibit negative refraction. Let us remark that, in addition to negative refraction, isotropic [39,40] and bianisotropic [41] chiral materials can support the allied phenomenon of negative reflection under certain conditions.

As regards notational matters: vectors are denoted by boldface with unit vectors being identified by an additional caret. Sans serif boldface indicates either a $3 \times 3$ dyadic or a $4 \times 4$ matrix, and the $3 \times 3$ identity dyadic is written as $\mathbf{I}$. The real and imaginary parts of complexvalued quantities are written as $\operatorname{Re}(\cdot)$ and $\operatorname{Im}(\cdot)$; the superscript * denotes the complex conjugate; and $i=\sqrt{-1}$. The permittivity and permeability of free space (i.e., vacuum) are written as $\epsilon_{0}$ and $\mu_{0}$, respectively, and $c_{0}=1 / \sqrt{\epsilon_{0} \mu_{0}}$. An $\exp (-i \omega t)$ dependence on time is implicit, where $\omega$ is the angular frequency and $t$ is time.

\section{Homogeneous and Homogenizable Chiral Materials}

\subsection{Preliminaries}

The characterization of negative refraction by chiral materials is considerably more complicated than of negative refraction by isotropic dielectric-magnetic materials, as more than one refraction wavevector needs to be considered as well as magnetoelectric coupling and possibly directionality [42]. Negative phase velocity (NPV) has been widely adopted as a convenient indicator of negative refraction $[43,44]$. While NPV and negative refraction go hand-in-hand for uniform planewave propagation in isotropic dielectric-magnetic materials, this is not generally true for nonuniform plane waves, especially in anisotropic materials. The possibility of NPV 
propagation in chiral materials was evident some years ago [45], but only nowadays is it being considered carefully [46-54].

Distinctions that can arise between negative refraction and NPV, as well as the closely related phenomenon of counterposition, in chiral metamaterials are highlighted in Sec. 2.5. Here we consider NPV propagation of uniform plane waves in certain well-known chiral materials and metamaterials.*

Let us consider electromagnetic field phasors

$$
\left.\begin{array}{l}
\mathbf{E}(\mathbf{r})=\mathbf{E}_{0} \exp (i k \hat{\mathbf{k}} \bullet \mathbf{r}) \\
\mathbf{H}(\mathbf{r})=\mathbf{H}_{0} \exp (i k \hat{\mathbf{k}} \bullet \mathbf{r})
\end{array}\right\},
$$

with complex-valued wavenumber $k$ and wavevector $\mathbf{k}=k \hat{\mathbf{k}}(|\hat{\mathbf{k}}|=1)$, in various types of homogeneous (or homogenizable) chiral materials. These materials are all included in the most general linear category of materials, namely bianisotropic materials, which are characterized by the frequency-domain constitutive relations [55]

$$
\left.\begin{array}{l}
\mathbf{D}(\mathbf{r})=\mathbf{\epsilon} \bullet \mathbf{E}(\mathbf{r})+\xi \bullet \mathbf{H}(\mathbf{r}) \\
\mathbf{B}(\mathbf{r})=\zeta \bullet \mathbf{E}(\mathbf{r})+\mu \bullet \mathbf{H}(\mathbf{r})
\end{array}\right\} .
$$

Combining Eqs. (1) and (2) with the source-free Maxwell curl postulates leads to the algebraic equation

$$
\mathbf{L}(\mathbf{k}) \bullet \mathbf{E}(\mathbf{r})=\mathbf{0},
$$

where the $3 \times 3$ dyadic

$$
\mathbf{L}(\mathbf{k})=(\mathbf{k} \times \mathbf{I}+\omega \xi) \cdot \boldsymbol{\mu}^{-1} \bullet(\mathbf{k} \times \mathbf{I}-\omega \zeta)+\omega^{2} \boldsymbol{\epsilon} .
$$

Solution of the dispersion relation

$$
\operatorname{det}[\mathbf{L}(\mathbf{k})]=0
$$

delivers the permissible wavenumbers. Since Eq. (5) represents a quartic polynomial, there are generally four wavenumbers, but the symmetries of the constitutive dyadics $\epsilon, \xi, \zeta$ and $\mu$ may result in the number of independent wavenumbers being less than four.

The phase velocity of the plane wave is classified as negative or positive, depending upon whether the scalar product $\operatorname{Re}(\mathbf{k}) \bullet \mathbf{P}(\mathbf{r})$ is negative or positive, where

$$
\mathbf{P}(\mathbf{r})=\frac{1}{2} \operatorname{Re}\left[\mathbf{E}(\mathbf{r}) \times \mathbf{H}^{*}(\mathbf{r})\right]
$$

is the time-averaged Poynting vector. As discussed in Sec. 2.5, the phase velocity can also be orthogonal to the time-averaged Poynting vector; i.e., $\operatorname{Re}(\mathbf{k}) \bullet \mathbf{P}(\mathbf{r})=0$. The ratio of $k$ to $k_{0}=\omega / c_{0}$ may be called a refractive index.

\subsection{Isotropic Chiral Materials}

The simplest chiral materials to consider are the isotropic chiral materials (ICMs). An everyday example of a homogeneous ICM is represented by an aqueous solution of dextrose at optical frequencies $[56,57]$. As another example, consider a random assembly of right-handed springs, each $\sim 2 \mathrm{~mm}$ long and $\sim 1 \mathrm{~mm}$ in diameter. While this assembly is nonhomogeneous at optical frequencies, it represents a homogenizable chiral material whose chirality is manifest under

*In Sec. 2.5 both uniform and nonuniform plane waves are considered. 


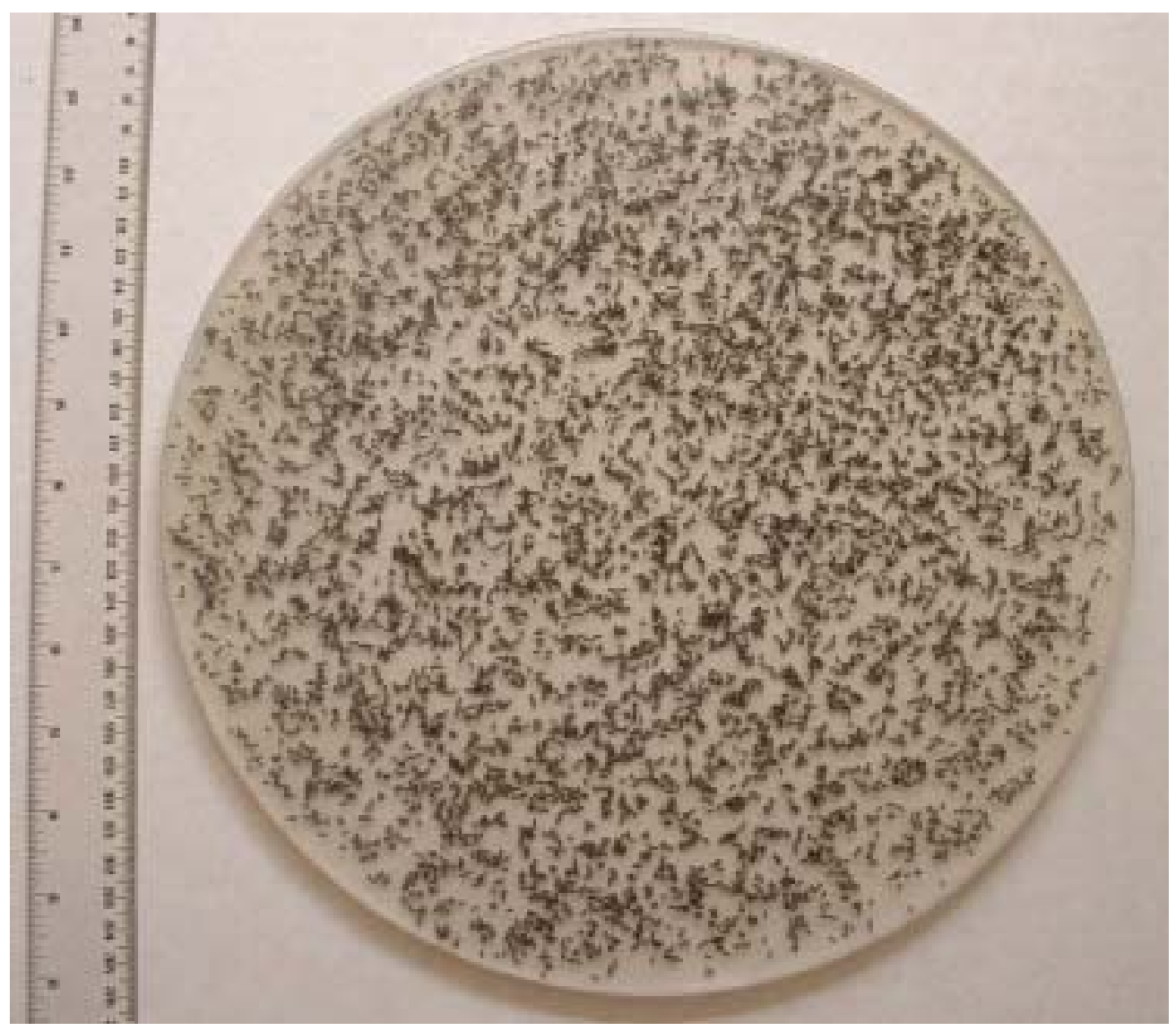

Fig. 3 An isotropic chiral material fabricated for microwave applications. Miniature springs are randomly dispersed in epoxy. (Courtesy of Álvaro Gómez, Universidad de Cantabria, Spain.)

microwave illumination [58-60]. Notice that the latter example represents a chiral metamaterial whereas the former does not. An illustration of an ICM fabricated for microwave applications is provided in Fig. 3.

Mathematically, ICMs are described by their frequency-domain constitutive relations [29]

$$
\left.\begin{array}{l}
\mathbf{D}(\mathbf{r})=\epsilon \mathbf{E}(\mathbf{r})+i \xi \mathbf{H}(\mathbf{r}) \\
\mathbf{B}(\mathbf{r})=-i \xi \mathbf{E}(\mathbf{r})+\mu \mathbf{H}(\mathbf{r})
\end{array}\right\}
$$

where $\xi$ is the chirality parameter. Equation (5) delivers four possible wavenumbers, $k=$ $k_{I C M}^{(1)}, k_{I C M}^{(2)}, k_{I C M}^{(3)}$, and $k_{I C M}^{(4)}$, where

$$
\left.\begin{array}{l}
k_{I C M}^{(1)}=-\omega(\sqrt{\epsilon \mu}+\xi) \\
k_{I C M}^{(2)}=\omega(\sqrt{\epsilon \mu}-\xi) \\
k_{I C M}^{(3)}=-k_{I C M}^{(2)} \\
k_{I C M}^{(4)}=-k_{I C M}^{(1)}
\end{array}\right\} .
$$


If the inequalities

$$
\left.\begin{array}{l}
\operatorname{Re}(\sqrt{\epsilon \mu}+\xi) \times \operatorname{Re}\left(\sqrt{\frac{\epsilon^{*}}{\mu^{*}}}\right)<0 \text { for } k=k_{I C M}^{(1),(4)} \\
\operatorname{Re}(\sqrt{\epsilon \mu}-\xi) \times \operatorname{Re}\left(\sqrt{\frac{\epsilon^{*}}{\mu^{*}}}\right)<0 \text { for } k=k_{I C M}^{(2),(3)}
\end{array}\right\}
$$

are satisfied, then NPV propagation is supported [24]. For a nondissipative isotropic chiral material (i.e., $\epsilon, \xi, \mu \in \mathbb{R}$ ) with $k_{I C M}^{(1),(2),(3),(4)} \in \mathbb{R}$, the NPV conditions [Eqs. (9)] reduce to

$$
\left.\begin{array}{c}
\sqrt{\epsilon \mu}<-\xi \quad \text { for } \quad k=k_{I C M}^{(1),(4)} \\
\sqrt{\epsilon \mu}<\xi \quad \text { for } \quad k=k_{I C M}^{(2),(3)}
\end{array}\right\} .
$$

Thus, we observe that NPV propagation arises only when the magnitude of the chirality parameter $\xi$ is sufficiently large relative to the magnitude of $\sqrt{\epsilon \mu}[24,61]$. Furthermore, the phase velocity can be positive for the plane waves with wavenumbers $k_{I C M}^{(1),(4)}$ but negative for the plane waves with wavenumbers $k_{I C M}^{(2),(3)}$, or vice versa [62]. The boundary between positive and negative phase velocity is characterized by infinite phase velocity [63].

The NPV conditions in Eqs. (9) and (10) highlight a key difference between ICMs and isotropic dielectric-magnetic materials. For the latter (which are described by the constitutive relations in Eq. (7) but with $\xi=0$ ), NPV arises when the inequality

$$
\frac{\operatorname{Re}(\epsilon)}{\operatorname{Im}(\epsilon)}+\frac{\operatorname{Re}(\mu)}{\operatorname{Im}(\mu)}<0
$$

is satisfied [44]. For passive isotropic dielectric-magnetic materials, a sufficient set of conditions for NPV is therefore $\operatorname{Re}(\epsilon)<0$ and $\operatorname{Re}(\mu)<0$. In practice, $\operatorname{Re}(\epsilon)<0$ may be achieved using metallic materials and $\operatorname{Re}(\mu)<0$ may be achieved using a microstructure based on split-ring resonators (SRRs), but it proves to be tricky to satisfy both of these conditions at the same frequency [20]. The introduction of chirality enables NPV to achieved without it being necessary for Eq. (11) (or $\operatorname{Re}(\epsilon)<0$ and $\operatorname{Re}(\mu)<0$ ) to be met. Furthermore, relatively strong chiral effects may be realized provided that the magnitude of $\sqrt{\epsilon \mu}$ is close to zero. A recent example is represented by the chiral metamaterial developed by Wang et al. [54], which exhibits a negative refractive index in the gigahertz frequency range. As shown in Fig. 4, their metamaterial is constructed from SRRs that are arranged on a cubic Bravais lattice. Theoretical and experimental studies confirm that the real part of the refractive index for one wavenumber is negative-valued, as illustrated in Fig. 5.

The prospects of $|\sqrt{\epsilon \mu}|$ being close to zero, and thereby satisfying the conditions for NPV, may be enhanced through exploiting materials with either $|\epsilon| \approx 0$ or $|\mu| \approx 0$ [64]. Such "epsilonnear-zero" metamaterials have recently been considered for antenna [65], imaging [66], and tunneling [67] applications, whereas "mu-near-zero" metamaterials have been considered for cloaking applications [68]. The canonical case of "epsilon-near-zero" and "mu-near-zero" for achiral materials is represented by nihility [69], wherein $\epsilon=\mu=0$. By restricting the notion of nihility to a particular frequency regime, and incorporating a nonzero chirality parameter $\xi$, the notion of chiral nihility emerges [21].

\subsection{Faraday Chiral Materials}

Suppose we have a random mixture of (i) an isotropic chiral material [29], as described by the frequency-domain constitutive relations in Eqs. (7), and (ii) either a magnetically biased 


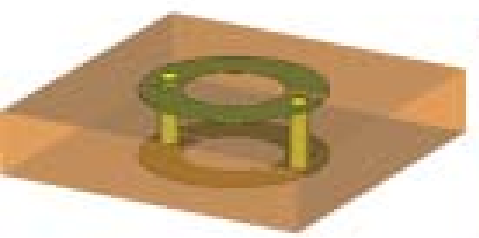

(a)

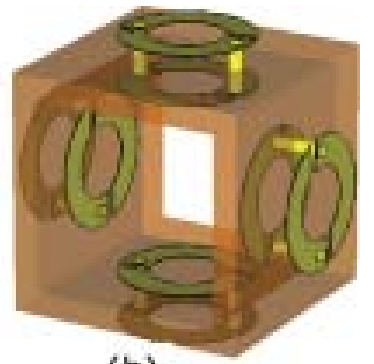

(b)

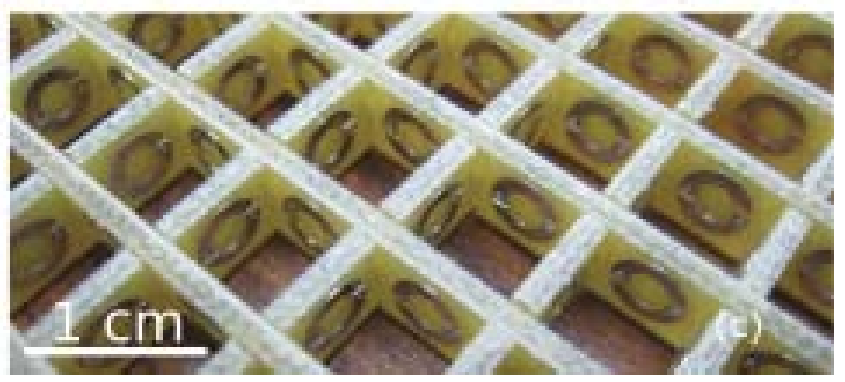

Fig. 4 The chiral metamaterial of Wang et al. [54]. Four split-ring resonators (a) are locked together to form the unit cell (b); and these unit cells are then arranged on a cubic lattice (c). (Reprinted with permission from Ref. 54. Copyright 2009 by the American Institute of Physics.)

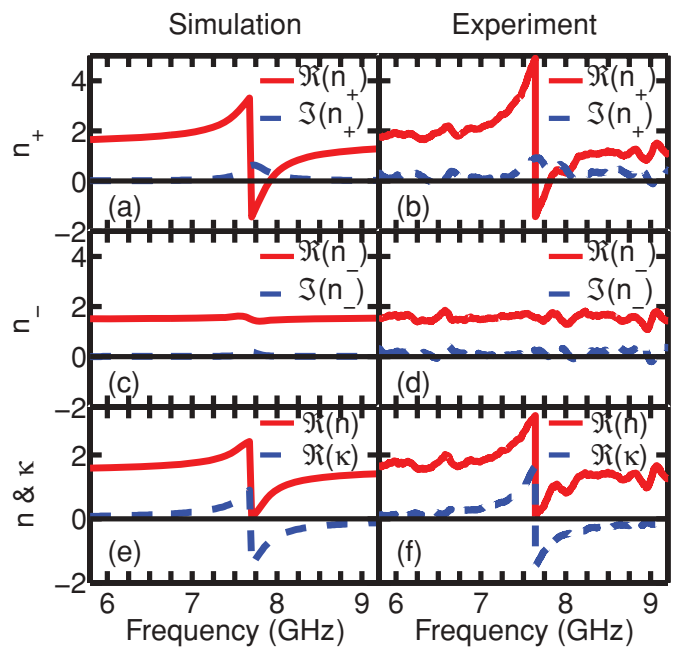

Fig. 5 Theoretical and experimental values of refractive indexes for the chiral metamaterial of Wang et al. [54] at gigahertz frequencies. Here, $\kappa=c_{0} \xi$ and $n=c_{0} \sqrt{\epsilon \mu}$, with $n_{ \pm}=n \pm \kappa$ being the refractive indexes for left- and right-handed waves. According to both theory and experiment, the real part of the index $n_{+}$is negative at $7.7 \mathrm{GHz}$. (Reprinted with permission from Ref. 54. Copyright 2009 by the American Institute of Physics.)

plasma [70], as described by the frequency-domain constitutive relations

$$
\left.\begin{array}{l}
\mathbf{D}(\mathbf{r})=\boldsymbol{\epsilon}_{\text {gyro }} \bullet \mathbf{E}(\mathbf{r}) \\
\mathbf{B}(\mathbf{r})=\mu_{0} \mathbf{H}(\mathbf{r})
\end{array}\right\},
$$

or a magnetically-biased ferrite [71], as described by the frequency-domain constitutive relations

$$
\left.\begin{array}{l}
\mathbf{D}(\mathbf{r})=\epsilon_{0} \mathbf{E}(\mathbf{r}) \\
\mathbf{B}(\mathbf{r})=\mu_{g y r o} \bullet \mathbf{H}(\mathbf{r})
\end{array}\right\},
$$


wherein the $3 \times 3$ constitutive dyadics have the gyrotropic form

$$
\boldsymbol{\tau}_{\text {gyro }}=\tau \mathbf{I}-i \tau_{g} \hat{\mathbf{u}} \times \mathbf{I}+\left(\tau_{u}-\tau\right) \hat{\mathbf{u}} \hat{\mathbf{u}}, \quad(\tau=\epsilon, \mu) .
$$

The unit vector $\hat{\mathbf{u}}$ identifies the direction of the biasing magnetic field. The mixture may be regarded as effectively homogeneous provided that wavelengths are much longer than the nonhomogeneities of the mixture. The resulting homogenized composite material (HCM) - which is known as a Faraday chiral material - combines natural optical activity, as exhibited by the isotropic chiral constituent material, with Faraday rotation, as exhibited by the gyrotropic constituent material [72].

Provided that the constituent materials are envisioned as electrically small spherical particles, the Faraday chiral material is described by the frequency-domain constitutive relations [73]

$$
\left.\begin{array}{l}
\mathbf{D}(\mathbf{r})=\mathbf{\epsilon}^{H C M} \bullet \mathbf{E}(\mathbf{r})+i \xi^{H C M} \bullet \mathbf{H}(\mathbf{r}) \\
\mathbf{B}(\mathbf{r})=-i \xi^{H C M} \bullet \mathbf{E}(\mathbf{r})+\boldsymbol{\mu}^{H C M} \bullet \mathbf{H}(\mathbf{r})
\end{array}\right\},
$$

where the three $3 \times 3$ constitutive dyadics have the common form

$$
\boldsymbol{\tau}^{H C M}=\tau^{H C M} \mathbf{I}-i \tau_{g}^{H C M} \hat{\mathbf{u}} \times \mathbf{I}+\left(\tau_{u}^{H C M}-\tau^{H C M}\right) \hat{\mathbf{u}} \hat{\mathbf{u}}, \quad(\tau=\epsilon, \xi, \mu) .
$$

More general Faraday chiral materials can arise through the homogenization of composite materials comprising nonspherical particles [74,75].

The constitutive properties of such HCMs can be estimated using a homogenization formalism. While many different formalisms have been developed [3], the strong-property-fluctuation theory $[76,77]$ provides the basis for a particularly sophisticated homogenization formalism that has been developed for isotropic [78] and anisotropic [75] chiral HCMs. As well as the requirement that the constituent particles of a composite material be electrically small $[79,80]$, the implementation of homogenization formalisms requires that the particles are numerous and distributed statistically uniformly throughout the composite material. Therefore, claims of negative refraction in a chiral metamaterial based on the homogenization of a composite material containing a single chiral inclusion must be regarded warily [81]. In a similar vein, the notion of homogenization precludes the possibility of the constituent materials containing resonant inclusions. Accordingly, claims of a negatively refracting chiral metamaterial arising from the homogenization of resonant inclusions must be viewed with caution too [82]. At best, one can obtain the constitutive parameters of an equivalent homogeneous medium that can replicate the experimental and/or numerical results obtained for a specific source-scatterer-detector configuration [83], but there is no guarantee that those constitutive parameters would be acceptable if the location of the source, the location of the detector, and/or the shape and size of the scatterer were to change without alteration of the composite material that the scatterer is made of.

Planewave propagation in Faraday chiral materials is rather more complicated than it is for isotropic chiral materials, because the effects of directionality come into play. For definiteness, let us choose the unit vector $\hat{\mathbf{u}}=\hat{\mathbf{z}}$ and consider propagation in the $x z$ plane; i.e.,

$$
\hat{\mathbf{k}}=\hat{\mathbf{x}} \sin \theta+\hat{\mathbf{z}} \cos \theta .
$$

Equation (5) then has the form [25]

$$
a_{4}\left(\frac{k}{\omega}\right)^{4}+a_{3}\left(\frac{k}{\omega}\right)^{3}+a_{2}\left(\frac{k}{\omega}\right)^{2}+a_{1}\left(\frac{k}{\omega}\right)+a_{0}=0
$$

with coefficients

$$
a_{4}=\left(\epsilon \sin ^{2} \theta+\epsilon_{z} \cos ^{2} \theta\right)\left(\mu \sin ^{2} \theta+\mu_{z} \cos ^{2} \theta\right)-\left(\xi \sin ^{2} \theta+\xi_{z} \cos ^{2} \theta\right)^{2},
$$




$$
\begin{aligned}
a_{3}= & 2 \cos \theta\left\{\sin ^{2} \theta\left[\mu_{g}\left(\epsilon \xi_{z}-\epsilon_{z} \xi\right)+\epsilon_{g}\left(\mu \xi_{z}-\mu_{z} \xi\right)+\xi_{g}\left(\mu \epsilon_{z}+\epsilon \mu_{z}-2 \xi \xi_{g}\right)\right]\right. \\
& \left.+2 \cos ^{2} \theta \xi_{g}\left(\epsilon_{z} \mu_{z}-\xi_{z}^{2}\right)\right\}, \\
a_{2}= & \sin ^{2} \theta\left\{\mu \mu_{z}\left(\epsilon_{g}^{2}-\epsilon^{2}\right)+\left(\xi^{2}+\xi_{g}^{2}\right)\left(\mu \epsilon_{z}+\epsilon \mu_{z}\right)-2 \xi\left[\xi_{z}\left(\xi_{g}^{2}-\xi^{2}\right)+\mu_{g} \epsilon_{z} \xi_{g}\right]\right. \\
& \left.-2 \epsilon_{g}\left[\xi_{z}\left(\mu_{g} \xi-\mu \xi_{g}\right)+\mu_{z} \xi \xi_{g}\right]-\epsilon\left[\epsilon_{z}\left(\mu^{2}-\mu_{g}^{2}\right)+2 \xi_{z}\left(\mu \xi-\mu_{g} \xi_{g}\right)\right]\right\} \\
& +2 \cos ^{2} \theta\left(\epsilon_{z} \mu_{z}-\xi_{z}^{2}\right)\left(3 \xi_{g}^{2}-\xi^{2}-\epsilon_{g} \mu_{g}-\epsilon \mu\right), \\
& a_{1}=4 \cos \theta\left(\epsilon_{z} \mu_{z}-\xi_{z}^{2}\right)\left[\xi\left(\epsilon_{g} \mu+\epsilon \mu_{g}\right)+\xi_{g}\left(\xi_{g}^{2}-\xi^{2}-\epsilon \mu-\epsilon_{g} \mu_{g}\right)\right], \\
a_{0}= & \left(\epsilon_{z} \mu_{z}-\xi_{z}^{2}\right)\left[\left(\epsilon^{2}-\epsilon_{g}^{2}\right)\left(\mu^{2}-\mu_{g}^{2}\right)+\left(\xi_{g}^{2}-\xi^{2}\right)^{2}-2\left(\xi_{g}^{2}+\xi^{2}\right)\left(\epsilon \mu+\epsilon_{g} \mu_{g}\right)\right. \\
& \left.+4 \xi \xi_{g}\left(\epsilon \mu_{g}+\mu \epsilon_{g}\right)\right] .
\end{aligned}
$$

Hence, four independent wavenumbers $k=k_{F C M}^{(1)}, k_{F C M}^{(2)}, k_{F C M}^{(3)}$, and $k_{F C M}^{(4)}$ may be extracted as the roots of Eq. (18). Propagation with NPV is supported provided that the real-valued scalar $W_{F C M}<0$, where

$$
\begin{aligned}
W_{F C M}= & \operatorname{Re}(k) \operatorname{Re}\left[\frac{1}{\mu_{z}^{*}}\left(\tilde{k}^{*} \sin \theta-i \xi_{z}^{*} \beta^{*}\right) \sin \theta\right. \\
& +\frac{1}{\left(\mu^{*}\right)^{2}-\left(\mu_{g}^{*}\right)^{2}}\left(\tilde { k } ^ { * } \left\{\left[\mu^{*}\left(|\alpha|^{2}+1\right)+i \mu_{g}^{*}\left(\alpha-\alpha^{*}\right)\right] \cos ^{2} \theta\right.\right. \\
& \left.+\mu^{*}|\beta|^{2} \sin ^{2} \theta-\left[\mu^{*}\left(\alpha^{*} \beta+\alpha \beta^{*}\right)+i \mu_{g}^{*}\left(\beta-\beta^{*}\right)\right] \sin \theta \cos \theta\right\} \\
& +\left(\mu^{*} \xi_{g}^{*}-\mu_{g}^{*} \xi^{*}\right)\left[\left(|\alpha|^{2}+1\right) \cos \theta-\alpha^{*} \beta \sin \theta\right] \\
& \left.\left.-i\left(\mu^{*} \xi^{*}-\mu_{g}^{*} \xi_{g}^{*}\right)\left[\left(\alpha-\alpha^{*}\right) \cos \theta-\beta \sin \theta\right]\right)\right]
\end{aligned}
$$

and $\alpha$ and $\beta$ are defined in terms of entries of $\mathbf{L}(\mathbf{k})$ per

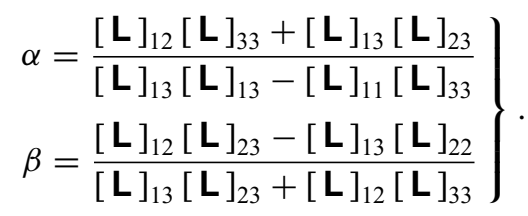

Matters simplify considerably when propagation is parallel to the $z$ axis (i.e., $\theta=0$ ). The four independent wavenumbers then reduce to

$$
\left.\begin{array}{l}
k_{F C M}^{(1)}=\omega\left(\sqrt{\epsilon+\epsilon_{g}} \sqrt{\mu+\mu_{g}}-\xi-\xi_{g}\right) \\
k_{F C M}^{(2)}=\omega\left(-\sqrt{\epsilon+\epsilon_{g}} \sqrt{\mu+\mu_{g}}-\xi-\xi_{g}\right) \\
k_{F C M}^{(3)}=\omega\left(\sqrt{\epsilon-\epsilon_{g}} \sqrt{\mu-\mu_{g}}+\xi-\xi_{g}\right) \\
k_{F C M}^{(4)}=\omega\left(-\sqrt{\epsilon-\epsilon_{g}} \sqrt{\mu-\mu_{g}}+\xi-\xi_{g}\right)
\end{array}\right\}
$$

and the NPV parameter $W_{F C M}$ for each wavenumber becomes

$$
W_{F C M}=2 \operatorname{Re}\left\{\sqrt{\epsilon+\epsilon_{g}} \sqrt{\mu+\mu_{g}}-\xi-\xi_{g}\right\} \operatorname{Re}\left\{\frac{\sqrt{\epsilon^{*}+\epsilon_{g}^{*}}}{\sqrt{\mu^{*}+\mu_{g}^{*}}}\right\} \quad \text { for } \quad k=k_{F C M}^{(1)},
$$




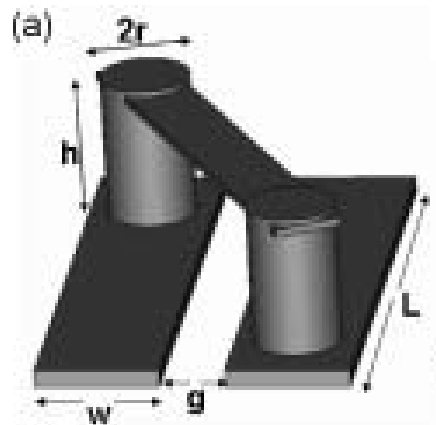

(c)

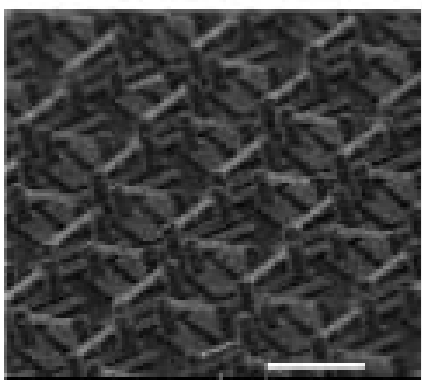

(b)

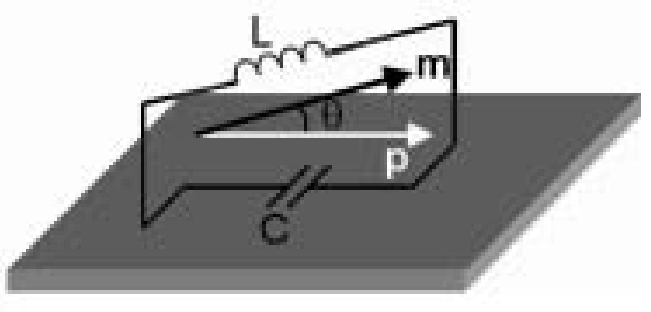

(d)

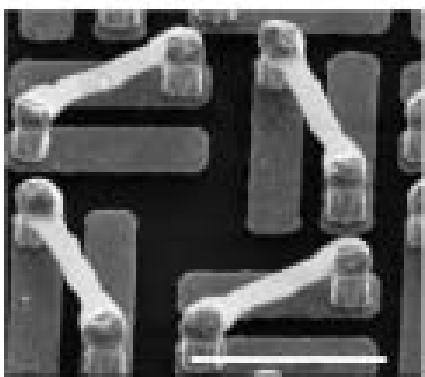

Fig. 6 The chiral metamaterial of Zhang et al. [84]. The microstructure comprises inductorcapacitor circuits (a) and (b). These are arranged in a tilted configuration to form the metamaterial (c) and (d). (Reprinted with permission from Ref. 84. Copyright 2009 by the American Physical Society.)

$$
\begin{aligned}
& W_{F C M}=2 \operatorname{Re}\left\{\sqrt{\epsilon+\epsilon_{g}} \sqrt{\mu+\mu_{g}}+\xi+\xi_{g}\right\} \operatorname{Re}\left\{\frac{\sqrt{\epsilon^{*}+\epsilon_{g}^{*}}}{\sqrt{\mu^{*}+\mu_{g}^{*}}}\right\} \quad \text { for } k=k_{F C M}^{(2)}, \\
& W_{F C M}=2 \operatorname{Re}\left\{\sqrt{\epsilon-\epsilon_{g}} \sqrt{\mu-\mu_{g}}+\xi-\xi_{g}\right\} \operatorname{Re}\left\{\frac{\sqrt{\epsilon^{*}-\epsilon_{g}^{*}}}{\sqrt{\mu^{*}-\mu_{g}^{*}}}\right\} \quad \text { for } k=k_{F C M}^{(3)}, \\
& W_{F C M}=2 \operatorname{Re}\left\{\sqrt{\epsilon-\epsilon_{g}} \sqrt{\mu-\mu_{g}}-\xi+\xi_{g}\right\} \operatorname{Re}\left\{\frac{\sqrt{\epsilon^{*}-\epsilon_{g}^{*}}}{\sqrt{\mu^{*}-\mu_{g}^{*}}}\right\} \quad \text { for } k=k_{F C M}^{(4)} .
\end{aligned}
$$

Numerical studies on the homogenization of an isotropic chiral material and a magnetically biased ferrite have revealed that the resulting Faraday chiral material can support NPV propagation provided that the gyrotropic constitutive parameter of the ferrite constituent material is sufficiently large in magnitude relative to the corresponding magnitudes of nongyrotropic permeability parameters [25]. In particular, Faraday chiral materials can support NPV propagation when their constituent materials do not.

A chiral metamaterial with constitutive relations very similar to those of the Faraday chiral material (15) has been developed recently by Zhang et al. [84]. As represented in Fig. 6, their metamaterial comprises metallic chiral resonators in which the loop of the resonator is tilted with respect to a reference plane. This metamaterial exhibits a negative-real refractive index for one wavenumber at frequencies between 1.06 and $1.27 \mathrm{THz}$, as shown in Fig. 7, despite the magnetic permeability having a positive real part over this frequency range. 
(3)

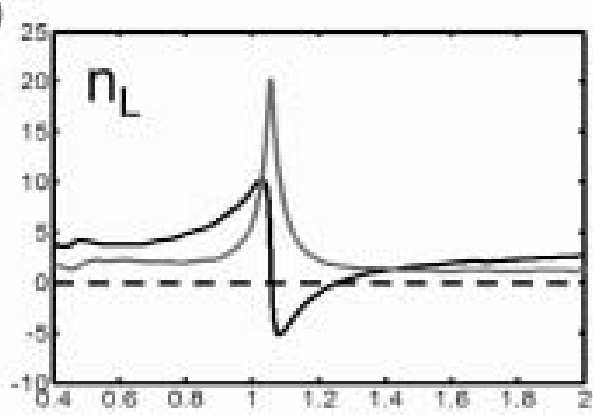

(c)

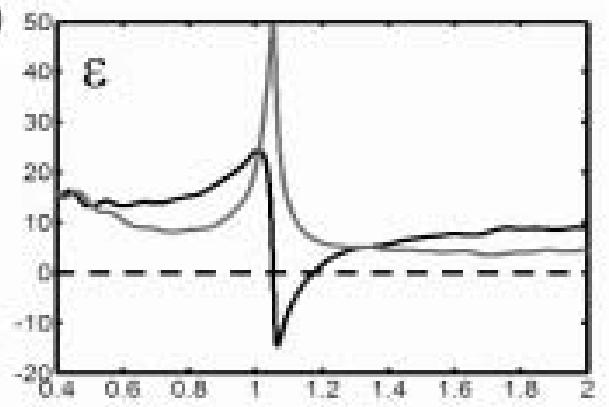

(b)

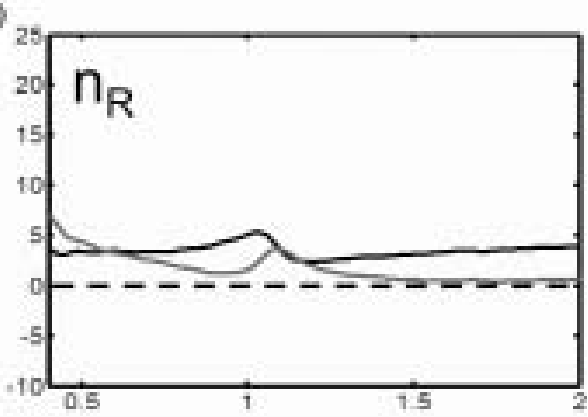

(d)

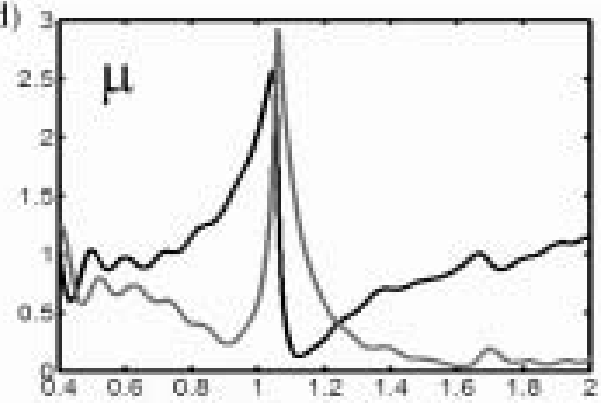

(e)

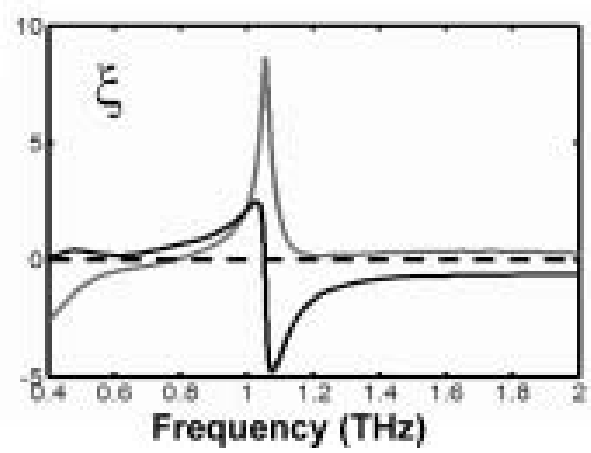

Fig. 7 Experimental values of refractive indexes ( $n_{L}$ for left-handed waves and $n_{R}$ for right-handed waves) for the chiral metamaterial of Zhang et al. [84], plotted against frequency (in terahertz). Also plotted are the dielectric, magnetic, and magnetoelectric constitutive parameters $\epsilon, \mu$, and $\xi$ respectively. Real parts are in black and imaginary parts are in gray. The real part of the index $n_{L}$ is negative between 1.06 and $1.27 \mathrm{THz}$. (Reprinted with permission from Ref. 84. Copyright 2009 by the American Physical Society.)

\subsection{Materials with Simultaneous Mirror-Conjugated and Racemic Chirality Characteristics}

Let us next consider an isotropic chiral material described by the constitutive relations in Eq. (7). The mirror conjugate material is also an isotropic chiral material, described by the constitutive relations in Eq. (7) but with the chirality parameter $\xi$ replaced by $-\xi$. When an isotropic chiral material and its mirror conjugate are mixed together in a random fashion, in equal proportions (and provided that no chemical reactions or molecular conformational changes occur), the resulting racemic mixture is an isotropic dielectric-magnetic material; i.e., it is achiral. However, an isotropic chiral material and its mirror conjugate can be mixed together in equal proportions and yet retain chirality, thereby producing a material with simultaneous mirror-conjugated and racemic chirality (SMCRC) characteristics. This can be achieved by fixing the orientations of the chiral elements that make up the constituents of the mixture. For example, an artificial SMCRC 
material may be conceptualized as a mixture of equal proportions of left-handed springs aligned along the $x$ axis and right-handed springs along the $y$ axis; and left- as well as right-handed springs in equal proportions aligned along the $z$ axis [85]; all springs are electrically small. Also, certain naturally occurring minerals exhibit SMCRC below their Néel temperatures $[86,87]$.

The $3 \times 3$ constitutive dyadics of an SMCRC material may be expressed as

$$
\left.\begin{array}{l}
\boldsymbol{\epsilon}=\epsilon_{1}(\hat{\mathbf{x}} \hat{\mathbf{x}}+\hat{\mathbf{y}} \hat{\mathbf{y}})+\epsilon_{3} \hat{\mathbf{z}} \hat{\mathbf{z}} \\
\xi=\xi(\hat{\mathbf{x}} \hat{\mathbf{x}}-\hat{\mathbf{y}} \hat{\mathbf{y}}) \\
\zeta=-\xi \\
\boldsymbol{\mu}=u_{1}(\hat{\mathbf{x}} \hat{\mathbf{x}}+\hat{\mathbf{y}} \hat{\mathbf{y}})+\mu_{3} \hat{\mathbf{z}} \hat{\mathbf{z}}
\end{array}\right\} .
$$

Equation (5) yields the four wavenumbers $k=k_{S M C R C}^{(1)}, k_{S M C R C}^{(2)}, k_{S M C R C}^{(3)}$, and $k_{S M C R C}^{(4)}$. However, since SMCRC materials are Lorentz-reciprocal [88], there are only two independent wavenumbers, at most. For propagation in an arbitrary direction given by

$$
\hat{\mathbf{k}}=\hat{\mathbf{x}} \sin \theta \cos \phi+\hat{\mathbf{y}} \sin \theta \sin \phi+\hat{\mathbf{z}} \cos \theta,
$$

the wavenumbers are [85]

$$
\left.\begin{array}{l}
k_{S M C R C}^{(1)}=\omega \sqrt{b_{3}\left(b_{1}-b_{2}\right)} \\
k_{S M C R C}^{(2)}=-k_{S M C R C}^{(1)} \\
k_{S M C R C}^{(3)}=\omega \sqrt{b_{3}\left(b_{1}+b_{2}\right)} \\
k_{S M C R C}^{(4)}=-k_{S M C R C}^{(3)}
\end{array}\right\}
$$

wherein the scalars

$$
\left.\begin{array}{l}
b_{1}=2 \epsilon_{z} \mu_{z} \cos ^{2} \theta+\left(\epsilon_{z} \mu+\epsilon \mu_{z}\right) \sin ^{2} \theta \\
b_{2}=\sin ^{2} \theta \sqrt{\left(\epsilon_{z} \mu-\epsilon \mu_{z}\right)^{2}-4 \epsilon_{z} \mu_{z} \xi^{2} \cos ^{2} 2 \phi} \\
b_{3}=\frac{1}{2}\left(\epsilon \mu+\xi^{2}\right)\left[\left(\epsilon \sin ^{2} \theta+\epsilon_{z} \cos ^{2} \theta\right)\left(\mu \sin ^{2} \theta+\mu_{z} \cos ^{2} \theta\right)+\xi^{2} \sin ^{4} \theta \cos ^{2} 2 \phi\right]^{-1}
\end{array}\right\}
$$

are introduced. Propagation of plane waves with NPV is supported provided that the real-valued scalar $W_{S M C R C}<0$, where [26]

$$
\begin{aligned}
W_{S M C R C}= & \operatorname{Re}(k)\left(\frac { 1 } { \omega } \left\{\left(|\alpha|^{2}+|\beta|^{2}\right) \cos ^{2} \theta+\sin ^{2} \theta\right.\right. \\
& \left.-\left[\left(\alpha+\alpha^{*}\right) \cos \phi+\left(\beta+\beta^{*}\right) \sin \phi\right] \sin \theta \cos \theta\right\} \\
& \times \operatorname{Re}\left(\frac{k^{*}}{\mu_{1}^{*}}\right)+\frac{1}{\omega}(\alpha \sin \phi-\beta \cos \phi)\left(\alpha^{*} \sin \phi-\beta^{*} \cos \phi\right) \sin ^{2} \theta \operatorname{Re}\left(\frac{k^{*}}{\mu_{3}^{*}}\right) \\
& \left.+\sin \theta \operatorname{Re}\left[\left(\alpha^{*} \sin \phi+\beta^{*} \cos \phi\right) \frac{\xi^{*}}{\mu_{1}^{*}}\right]-\left(\alpha \beta^{*}+\alpha^{*} \beta\right) \cos \theta \operatorname{Re}\left(\frac{\xi^{*}}{\mu_{1}^{*}}\right)\right),
\end{aligned}
$$

and the scalars $\alpha$ and $\beta$ are defined as in Eqs. (25).

For planewave propagation along the racemic axis (i.e., $\hat{\mathbf{k}}=\hat{\mathbf{z}}$ ), only one independent wavenumber, namely

$$
k_{S M C R C}^{(1)}=-k_{S M C R C}^{(2)}=k_{S M C R C}^{(3)}=-k_{S M C R C}^{(4)}=\omega \sqrt{\epsilon_{1} \mu_{1}+\xi^{2}},
$$


emerges from Eq. (5). The corresponding NPV parameter $W_{S M C R C}$ simplifies as

$$
\begin{aligned}
W_{S M C R C}= & \left(\left|\frac{\alpha}{\beta}\right|^{2}+1\right) \operatorname{Re}\left[\frac{\left(\sqrt{\epsilon_{1} \mu_{1}+\xi^{2}}\right)^{*}}{\mu_{1}^{*}}\right] \\
& -2 \operatorname{Re}\left(\frac{\alpha}{\beta}\right) \operatorname{Re}\left(\frac{\xi^{*}}{\mu_{1}^{*}}\right) \operatorname{Re}\left(\sqrt{\epsilon_{1} \mu_{1}+\xi^{2}}\right) \quad \text { for } k=k_{S M C R C}^{(1),(3)}, \\
W_{S M C R C}= & \left(\left|\frac{\alpha}{\beta}\right|^{2}+1\right) \operatorname{Re}\left[\frac{\left(\sqrt{\epsilon_{1} \mu_{1}+\xi^{2}}\right)^{*}}{\mu_{1}^{*}}\right] \\
& +2 \operatorname{Re}\left(\frac{\alpha}{\beta}\right) \operatorname{Re}\left(\frac{\xi^{*}}{\mu_{1}^{*}}\right) \operatorname{Re}\left(\sqrt{\epsilon_{1} \mu_{1}+\xi^{2}}\right) \quad \text { for } k=k_{S M C R C}^{(2),(4)} .
\end{aligned}
$$

Numerical studies indicate that NPV arises as long as that the magnitude of the magnetoelectric constitutive parameter $\xi$ is sufficiently large compared with the magnitudes of dielectric and magnetic constitutive parameters [26]. In addition, the directions of NPV propagation are influenced little by $|\xi|$. Furthermore, the incorporation of dielectric and magnetic constitutive parameters with negative-real parts enhances the propensity for NPV propagation.

\subsection{Counterposition, Negative Phase Velocity, and Negative Refraction}

While NPV may be a useful indicator of the propensity for negative refraction by certain homogeneous materials, NPV can arise in the absence of negative refraction (and vice versa) in certain anisotropic [89] and bianisotropic [90] materials and metamaterials. Furthermore, from a practical point of view, the direction of energy flow, as provided by the time-averaged Poynting vector, may be more pertinent than the direction of the wavevector. Therefore, in the context of NPV and negative refraction, the phenomenon of counterposition should be considered too. This arises at a planar interface when the refraction wavevector and its associated time-averaged Poynting vector are oriented on opposite sides of the normal to the interface, as has been described for certain crystals [91,92].

In order to explore the relationships between counterposition, NPV and negative refraction, let us focus on the Lorentz-reciprocal [88] bianisotropic material—known as a pseudochiral omega material [93] — described by the constitutive dyadics [94]

$$
\left.\begin{array}{l}
\boldsymbol{\epsilon}=\epsilon_{x} \hat{\mathbf{x}} \hat{\mathbf{x}}+\epsilon_{y} \hat{\mathbf{y}} \hat{\mathbf{y}}+\epsilon_{z} \hat{\mathbf{z}} \hat{\mathbf{z}} \\
\xi=-i \xi \hat{\mathbf{z}} \hat{\mathbf{y}} \\
\zeta=i \xi \hat{\mathbf{y}} \mathbf{\mathbf { z }} \\
\boldsymbol{\mu}=\mu_{x} \hat{\mathbf{x}} \hat{\mathbf{x}}+\mu_{y} \hat{\mathbf{y}} \hat{\mathbf{y}}+\mu_{z} \hat{\mathbf{z}} \hat{\mathbf{z}}
\end{array}\right\} .
$$

These particular constitutive dyadics are used to describe a metamaterial assembled from layers of split-ring resonators [98], this being a commonplace configuration for metamaterials [99,100]. As well as NPV, such magnetoelectric metamaterials may support other exotic electromagnetic phenomenons, for example, longitudinal polarization [101].

Suppose that a metamaterial described by Eqs. (39) fills the half-space $z>0$, while the half-space $z<0$ is vacuous. The wave vectors of the incident, the reflected, and the refracted plane waves lie wholly in the $x z$ plane. The wavevectors for the two refracted plane waves that exist in the half-space $z>0$ may be expressed as

$$
\mathbf{k}^{(j)}=\kappa \hat{\mathbf{x}}+k_{z j} \hat{\mathbf{z}}, \quad(j=1,2),
$$




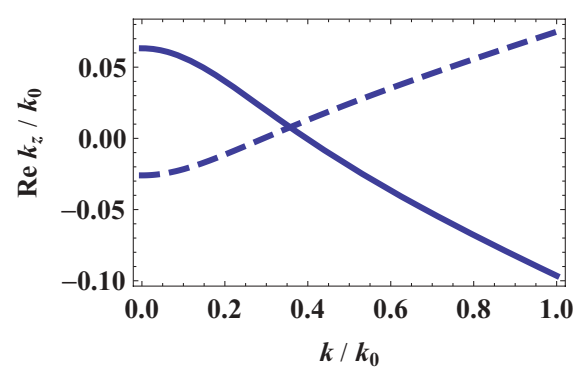

(a)

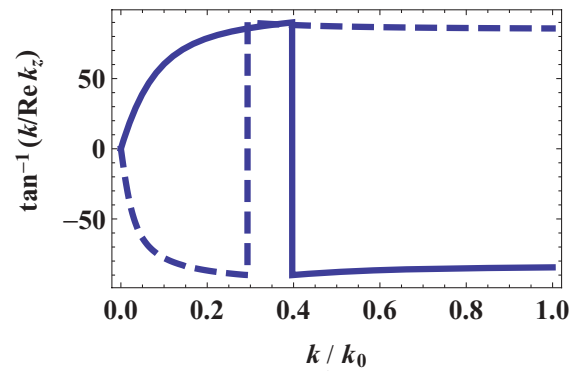

(b)

Fig. 8 (a) Real part of $k_{z j} / k_{0}$ and (b) the angle (in degrees) between the real part of $\mathbf{k}^{(j)}$ and the positive $z$ axis, both plotted against $\kappa / k_{0}$. The solid curves correspond to the plane wave labeled $j=1$, and the dashed curves to the plane wave labeled $j=2$. Note that $\operatorname{Im}\left(k_{z j}\right)>0$ for all $\kappa / k_{0} \in[0,1)$, for both values of $j$. See Sec. 2.5 for other details.

where the real-valued

$$
\kappa=k_{0} \sin \theta \in\left[0, k_{0}\right),
$$

with the free-space wavenumber $k_{0}=\omega \sqrt{\epsilon_{0} \mu_{0}}$. The wavevector components $k_{z j}$ are delivered from Eq. (5) as follows:

$$
\left.\begin{array}{l}
k_{z 1}=k_{0} \sqrt{\mu_{x}\left(\epsilon_{y}-\frac{\kappa^{2}}{\mu_{z} k_{0}^{2}}\right)} \\
k_{z 2}=k_{0} \sqrt{\frac{\epsilon_{x}}{\epsilon_{z}}\left[\left(\epsilon_{z} \mu_{y}-\xi^{2}\right)-\frac{\kappa^{2}}{k_{0}^{2}}\right]}
\end{array}\right\} .
$$

Herein, the square roots must be evaluated such that both refracted plane waves transport energy away from the interface $z=0$ in the half-space $z>0$. Since $k_{z j} \in \mathbb{C}$ in general, the refracted plane waves may be nonuniform. The time-averaged Poynting vector corresponding to the plane wave labeled as $j$ is written as $\mathbf{P}_{j}$.

Confusingly, the term negative refraction has been used to mean different things. The sense of refraction is determined solely by the relative orientations of the real parts of the refraction and incidence wavevectors, in keeping with Snel's law, which emanates from the principle of conservation of linear momentum. Thus, negative refraction arises whenever the real part of $k_{z 1}$ (or $k_{z 2}$ ) is negative. In contrast, negative refraction was also set up [89] as the negative "refraction of the energy flux density," but it amounts to a negative-deflection phenomenon that can occur whether refraction is positive or negative and is easily appreciated as such when the incident plane wave is replaced by a beam (of finite width). Similar comments apply to certain later works too [95-97].

In order to illustrate the distinctions between counterposition, NPV, and negative refraction, let us investigate a specific example in which the constitutive parameters for the bianisotropic material occupying the half-space $z>0$ are: $\epsilon_{x}=0.1+0.03 i, \epsilon_{y}=0.14+0.02 i, \epsilon_{z}=0.13+$ $0.07 i ; \mu_{x}=-0.2+0.2 i, \quad \mu_{y}=-0.22+0.03 i, \quad \mu_{z}=-0.27+0.6 i ;$ and $\xi=0.21+0.05 i$. This is a dissipative material.

The real part of $k_{z j} / k_{0},(j=1,2)$, is plotted as a function of $\left(\kappa / k_{0}\right) \in[0,1)$ in Fig. 8. Also presented in Fig. 8 are plots of the angle between the real part of $\mathbf{k}^{(j)},(j=1,2)$, and the positive $z$ axis. We see that the refracted plane wave labeled 1 is positively refracted for $0<\left(\kappa / k_{0}\right)<$ 0.4 but negatively refracted for $0.4<\left(\kappa / k_{0}\right)<1$; also, the refracted plane wave labeled 2 is negatively refracted for $0<\left(\kappa / k_{0}\right)<0.29$ but positively refracted for $0.29<\left(\kappa / k_{0}\right)<1$. 


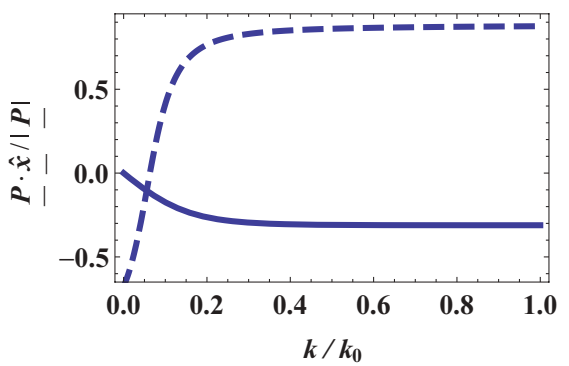

(a)

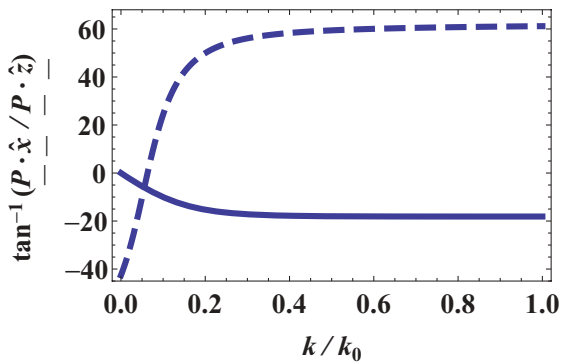

(b)

Fig. 9 (a) The $x$ component of the normalized time-averaged Poynting vector and (b) the angle (in degrees) between the time-averaged Poynting vector and the positive $z$ axis, both plotted against $\kappa / k_{0}$. The solid curves correspond to the plane wave labeled $j=1$, and the dashed curves to the plane wave labeled $j=2$. Note that $\hat{\mathbf{z}} \bullet \mathbf{P}>0$ for all $\kappa / k_{0} \in[0,1)$, for both plane waves. See Sec. 2.5 for other details.

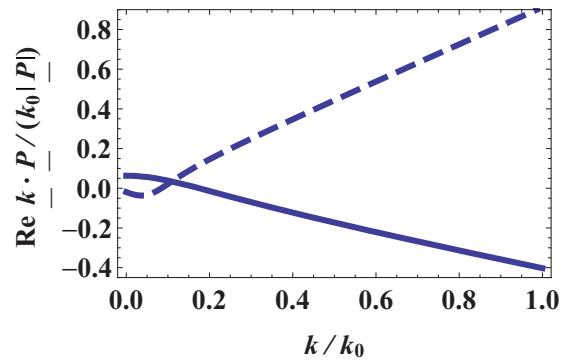

Fig. 10 The quantity $\operatorname{Re}\left(\mathbf{k}^{(j)}\right) \bullet \mathbf{P}_{j} /\left(k_{0}\left|\mathbf{P}_{j}\right|\right)$ plotted against $\left(\kappa / k_{0}\right) \in[0,1)$. The solid curve corresponds to the plane wave labeled $j=1$, and the dashed curve to the plane wave labeled $j=2$. See Sec. 2.5 for other details.

Since the imaginary parts of both $k_{z 1}$ and $k_{z 2}$ are positive, both refracted plane waves attenuate as $z \rightarrow \infty$.

In Fig. 9, the normalized $x$ component of the time-averaged Poynting vectors for both refracted plane waves are plotted against $\left(\kappa / k_{0}\right)$. Also plotted is the angle between $\mathbf{P}_{j}$ and the positive $z$ axis, for both refracted plane waves. The $x$ component of $\mathbf{P}_{1}$ is negative for all $\left(\kappa / k_{0}\right) \in[0,1)$, but the $x$ component of $\mathbf{P}_{2}$ is negative only for $\left(\kappa / k_{0}\right) \in[0,0.06)$. That is, $\mathbf{P}_{1}$ always subtends a negative angle to the positive $z$ axis while the sign of the angle that $\mathbf{P}_{2}$ subtends depends on $\kappa$. The $z$ components of $\mathbf{P}_{j}$ for both refracted plane waves are positive for all $\left(\kappa / k_{0}\right) \in[0,1)$, as is required by the choice of square roots in Eq. (42).

When the inequality

$$
\operatorname{Re}\left(k_{z j}\right) \hat{\mathbf{x}} \bullet \mathbf{P}_{j}<0
$$

is satisfied, counterposition occurs [92]. From Figs. 8 and 9, it may be inferred that counterposition occurs for the refracted plane wave labeled 1 when $0<\left(\kappa / k_{0}\right)<0.4$. Hence, the $\kappa$-range for positive refraction is the same as that for counterposition for the planewave labeled 1 . This contrasts with the situation for the plane wave labeled 2 where counterposition arises only for $0.06<\left(\kappa / k_{0}\right)<0.29$.

The sign of the phase velocity for the plane wave labeled $j$ is determined the quantity $\operatorname{Re}\left(\mathbf{k}^{(j)}\right) \bullet \mathbf{P}_{j}$, which is plotted against $\left(\kappa / k_{0}\right) \in[0,1)$ in Fig. 10. The refracted plane wave labeled 1 has NPV for $0.18<\left(\kappa / k_{0}\right)<1$, whereas NPV is exhibited by the refracted plane wave labeled 2 for $0<\left(\kappa / k_{0}\right)<0.08$. Notice that the $\kappa$-ranges for NPV and negative refraction do not coincide. Take, for example, $\kappa=0.2 k_{0}$ : (i) The angle between $\operatorname{Re}\left(\mathbf{k}^{(1)}\right)$ and the $+z$ axis is $79 \mathrm{deg}$ and the angle between $\mathbf{P}_{1}$ and the $+z$ axis is $-15 \mathrm{deg}$. Thus, here the plane wave is 
Table 1 Values of $\kappa$ for which negative/positive refraction, counterposition, and negative/positive/orthogonal phase velocity occur for the plane wave labeled 1 . The right-most column shows the directions in the half-space $z>0$ of $\operatorname{Re}\left(\mathbf{k}^{(1)}\right)$ (thick dashed arrows) and $\mathbf{P}_{1}$ (thick solid arrows) for representative values of $\kappa$; also shown are the directions of the wavevectors for the incident and reflected plane waves in the half-space $z<0$ (thin solid arrows).

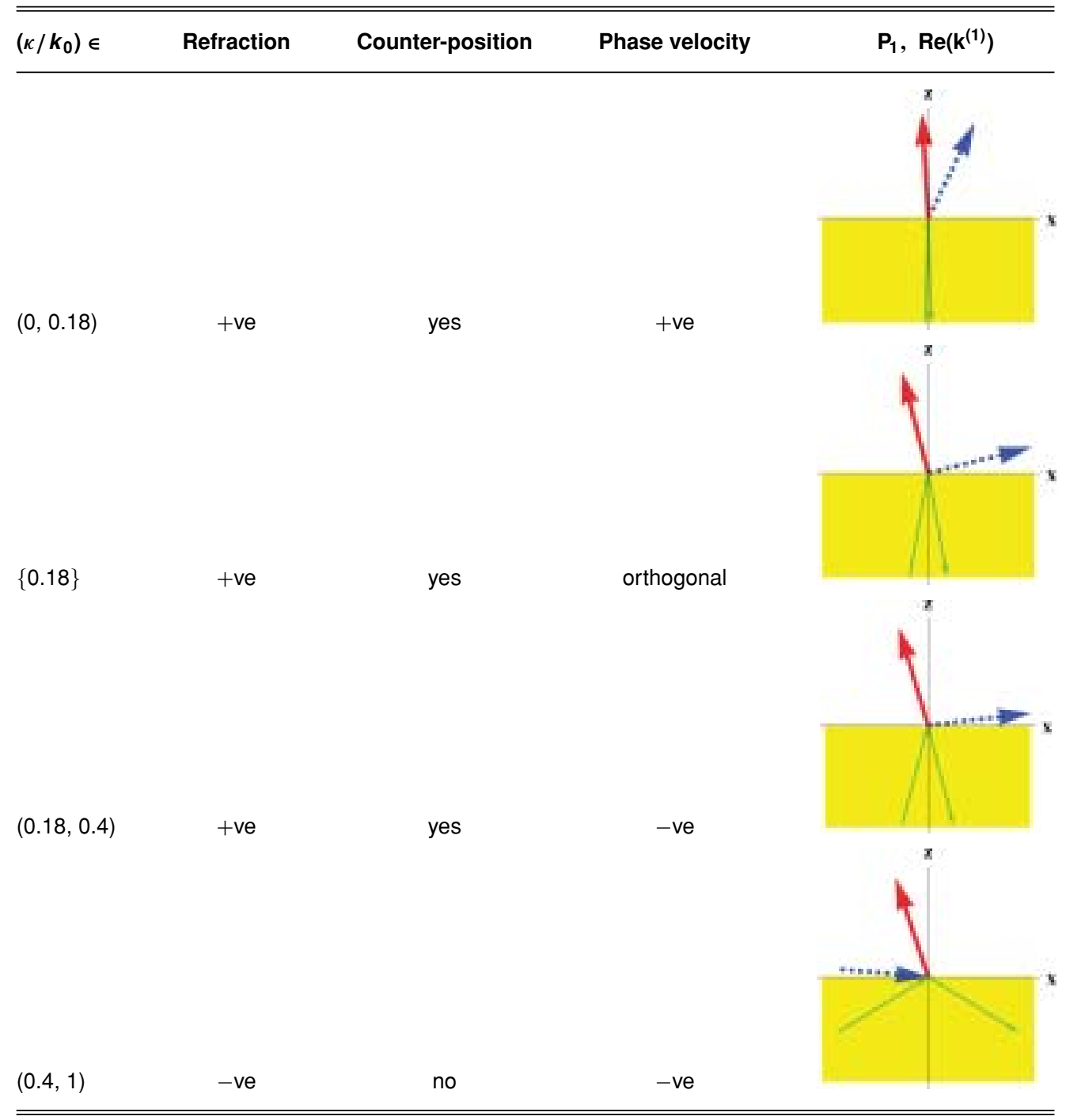

positively refracted but it has NPV. (ii) The angle between $\operatorname{Re}\left(\mathbf{k}^{(2)}\right)$ and the $+z$ axis is $-87 \mathrm{deg}$ and the angle between $\mathbf{P}_{2}$ and the $+z$ axis is $50 \mathrm{deg}$. This plane wave is negatively refracted despite having positive phase velocity.

Exceptionally, the phase velocity can be neither positive nor negative. At $\kappa=0.18 k_{0}$, the phase velocity vector and the time-averaged Poynting vector are orthogonal for the plane wave labeled 1 . Orthogonal phase velocity occurs for the plane wave labeled 2 at $\kappa=0.08 k_{0}$.

The foregoing results are summarized in Tables 1 and 2 for the plane waves labeled 1 and 2, respectively. Included in these tables are illustrations of the directions of $\operatorname{Re}\left(\mathbf{k}^{(j)}\right)$ and $\mathbf{P}_{j}(j=1,2)$, for representative values of $\kappa$. This numerical example illustrates the complexity that can arise at the planar boundary of a chiral metamaterial — indeed, the independence of negative refraction and NPV can even arise for isotropic dielectric materials if nonuniform plane waves are taken into consideration [90]. 
Mackay and Lakhtakia: Negatively refracting chiral metamaterials...

Table 2 As Table 1 but for the plane wave labeled 2.

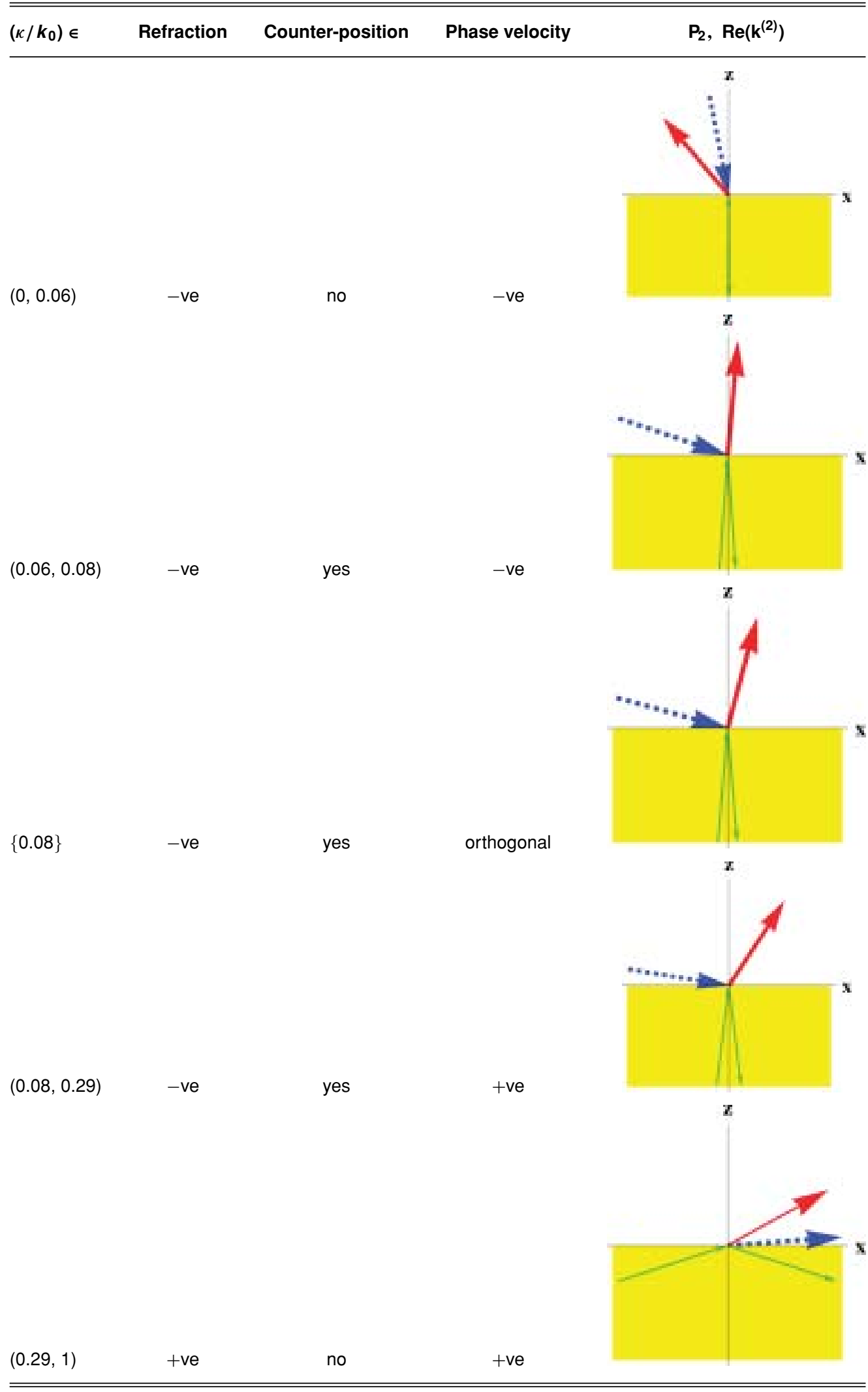




\section{Structurally Chiral Materials}

\subsection{Preliminaries}

In contrast to the materials considered in Sec. 2, structurally chiral materials owe their chirality to the handedness of their morphology at macroscopic length scales (i.e., at length scales comparable to the wavelength). A very general class of structurally chiral materials is represented by helicoidal bianisotropic materials (HBMs). These include chiral nematic and chiral smectic liquid crystals as well as sculptured thin films. The nonhomogeneity of HBMs - which gives rise to their chirality - is spatially continuous at macroscopic length scales. Another technologically important type of structurally chiral material is represented by ambichiral materials. These are piecewise homogeneous in a certain direction [32]. The propensity of both HBMs and ambichiral materials to support negative refraction is reviewed in the following sections.

\subsection{Helicoidal Bianisotropic Materials}

The frequency-domain constitutive relations [102]

$$
\left.\begin{array}{l}
\mathbf{D}(\mathbf{r})=\boldsymbol{\epsilon}_{H B M}(z) \bullet \mathbf{E}(\mathbf{r})+\xi_{H B M}(z) \bullet \mathbf{H}(\mathbf{r}) \\
\mathbf{B}(\mathbf{r})=\zeta_{H B M}(z) \bullet \mathbf{E}(\mathbf{r})+\boldsymbol{\mu}_{H B M}(z) \bullet \mathbf{H}(\mathbf{r})
\end{array}\right\}
$$

describe a HBM with the $z$ axis being the helicoidal axis. The four $3 \times 3$ nonhomogeneous constitutive dyadics herein have the general form

$$
\beta_{H B M}(z)=\left(\begin{array}{lll}
\beta_{11}(z) & \beta_{12}(z) & \beta_{13}(z) \\
\beta_{21}(z) & \beta_{22}(z) & \beta_{23}(z) \\
\beta_{31}(z) & \beta_{32}(z) & \beta_{33}(z)
\end{array}\right), \quad(\beta=\epsilon, \xi, \zeta, \mu),
$$

and may be expressed as

$$
\boldsymbol{\beta}_{H B M}(z)=\mathbf{S}(z) \bullet \boldsymbol{\beta}_{H B M}(0) \bullet \mathbf{S}^{-1}(z), \quad(\beta=\epsilon, \xi, \zeta, \mu) .
$$

The helicoidal architecture is specified by the rotation dyadic

$$
\mathbf{S}(z)=\left(\begin{array}{ccc}
\cos \left(\frac{\pi z}{\Omega}\right) & -h \sin \left(\frac{\pi z}{\Omega}\right) & 0 \\
h \sin \left(\frac{\pi z}{\Omega}\right) & \cos \left(\frac{\pi z}{\Omega}\right) & 0 \\
0 & 0 & 1
\end{array}\right)
$$

with $\Omega$ being the half-period. The HBM is structurally right-handed if $h=1$, left-handed if $h=-1$, and structurally achiral if $h=0$.

A host of structurally chiral materials are encompassed by the constitutive Eqs. (44). The chirality may be purely dielectric, in which case $\xi_{H B M}=\zeta_{H B M}=\mathbf{0}$ and $\mu_{H B M}=\mu_{0} \mathbf{l}$. This subcategory includes chiral nematic liquid crystals (wherein $\epsilon_{H B M}$ has a uniaxial form), chiral smectic liquid crystals (wherein $\boldsymbol{\epsilon}_{H B M}$ has a biaxial form) [30,31]; and their solid-state counterparts called chiral sculptured thin films [33]. Illustrations of organic and inorganic chiral sculptured thin films, both fabricated by vapor deposition techniques, are provided in Fig. 11. The chirality may also be purely magnetic, in which case $\xi_{H B M}=\zeta_{H B M}=\mathbf{0}$ and $\boldsymbol{\epsilon}_{H B M}=\epsilon_{0} \mathbf{I}$. Fully bianisotropic chirality may be achieved through infiltrating a chiral sculptured thin film with an isotropic chiral fluid [104,105].

Most notably, HBMs can discriminate between left- and right-circularly polarized light. Let us consider a plane wave normally incident on a slab of an HBM that has its helicoidal axis 

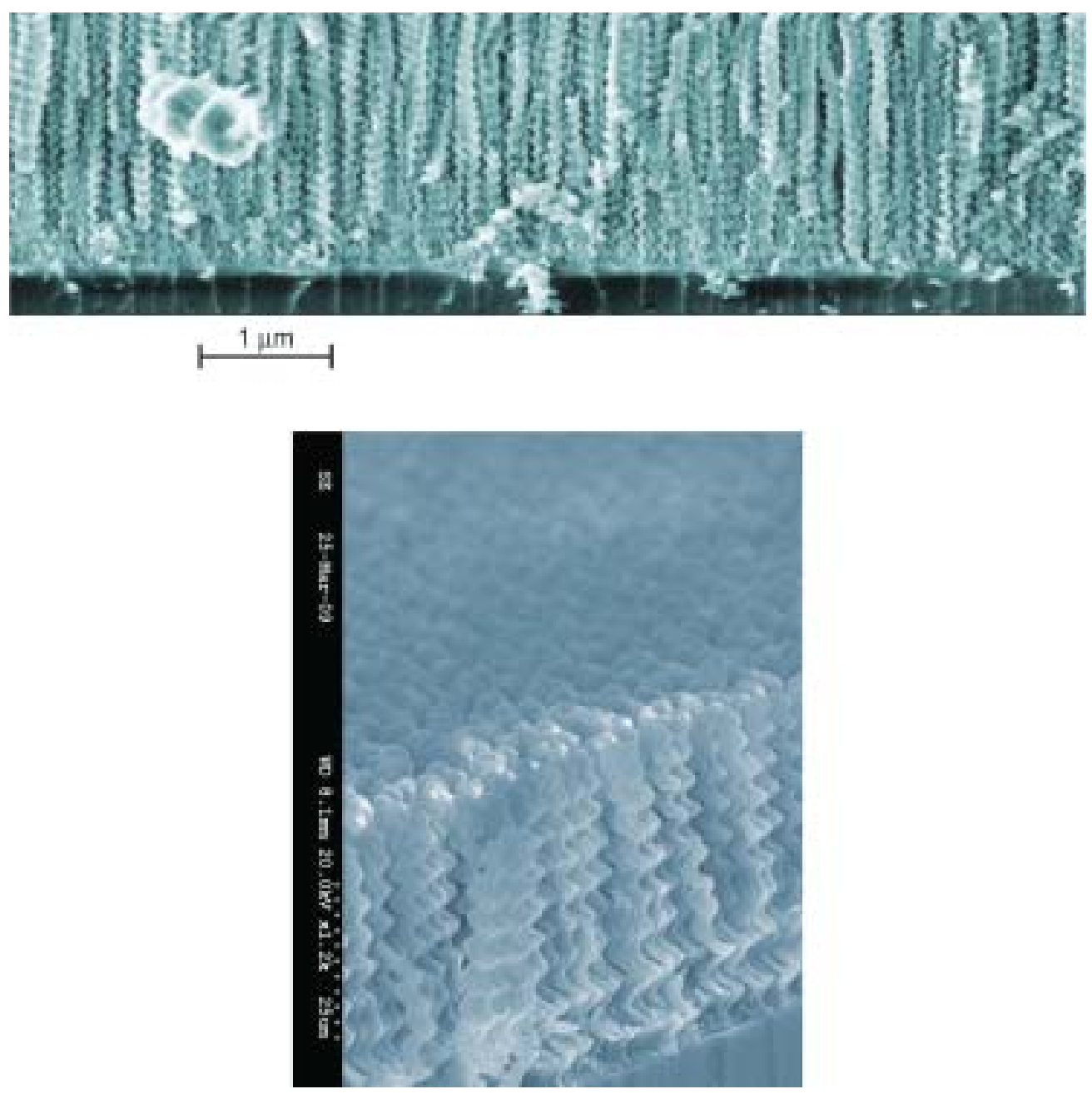

Fig. 11 Scanning electron microscope images of chiral sculptured thin films made from silicon oxide (top, Courtesy of Mark W. Horn, Pennsylvania State University) and parylene [103] (bottom).

aligned with the direction of slab thickness. Within a certain wavelength regime, the incident light may be almost entirely reflected if the structural handedness of the HBM coincides with the handedness of the incident light. Conversely, if the two handednesses are opposite then the incident light will undergo almost no reflection. This circular Bragg phenomenon underpins many actual and potential technological applications of HBMs [33].

The propagation of plane waves in HBMs has been comprehensively analyzed [102,106]. Combining the constitutive Eqs. (44) with the source-free Maxwell curl postulates yields the system of partial differential equations

$$
\left.\begin{array}{l}
\nabla \times \mathbf{E}(\mathbf{r})=i \omega\left[\zeta_{H B M}(z) \bullet \mathbf{E}(\mathbf{r})+\boldsymbol{\mu}_{H B M}(z) \bullet \mathbf{H}(\mathbf{r})\right] \\
\nabla \times \mathbf{H}(\mathbf{r})=-i \omega\left[\boldsymbol{\epsilon}_{H B M}(z) \bullet \mathbf{E}(\mathbf{r})+\xi_{H B M}(z) \bullet \mathbf{H}(\mathbf{r})\right]
\end{array}\right\} .
$$

Upon implementing the Oseen transformation [109]

$$
\left.\begin{array}{l}
\mathbf{E}^{\prime}(\mathbf{r})=\mathbf{S}^{-1}(z) \bullet \mathbf{E}(\mathbf{r}) \\
\mathbf{H}^{\prime}(\mathbf{r})=\mathbf{S}^{-1}(z) \bullet \mathbf{H}(\mathbf{r})
\end{array}\right\}
$$


and introducing the planewave representations

$$
\left.\begin{array}{l}
\mathbf{E}^{\prime}(\mathbf{r})=\mathbf{E}_{0}^{\prime}(z) \exp \left(i \kappa_{H B M} x\right) \\
\mathbf{H}^{\prime}(\mathbf{r})=\mathbf{H}_{0}^{\prime}(z) \exp \left(i \kappa_{H B M} x\right)
\end{array}\right\},
$$

wherein $\kappa_{H B M}$ may be interpreted as a wavenumber in the $x y$ plane, Eqs. (48) generally reduce to the $4 \times 4$ matrix differential equation

$$
\frac{\partial}{\partial z} \mathbf{F}_{0}^{\prime}(z)=\mathbf{M}^{\prime}(z) \bullet \mathbf{F}_{0}^{\prime}(z)
$$

Herein, the column 4-vector

$$
\mathbf{F}_{0}^{\prime}(z)=\left[\begin{array}{c}
\hat{\mathbf{x}} \bullet \mathbf{E}_{0}^{\prime}(z) \\
\hat{\mathbf{y}} \bullet \mathbf{E}_{0}^{\prime}(z) \\
\hat{\mathbf{x}} \bullet \mathbf{H}_{0}^{\prime}(z) \\
\hat{\mathbf{y}} \bullet \mathbf{H}_{0}^{\prime}(z)
\end{array}\right],
$$

and the $4 \times 4$ matrix function $\mathbf{M}^{\prime}(z)$ may be formally expressed as

$$
\begin{aligned}
\mathbf{M}^{\prime}(z)= & \mathbf{A}^{\prime}+\kappa_{H B M}\left[\mathbf{C}_{1,1}^{\prime} \exp \left(\frac{i \pi z}{\Omega}\right)+\mathbf{C}_{1,-1}^{\prime} \exp \left(-\frac{i \pi z}{\Omega}\right)\right] \\
& +\kappa_{H B M}^{2}\left[\mathbf{C}_{2,2}^{\prime} \exp \left(\frac{i 2 \pi z}{\Omega}\right)+\mathbf{C}_{2,0}^{\prime}+\mathbf{C}_{2,-2}^{\prime} \exp \left(-\frac{i 2 \pi z}{\Omega}\right)\right],
\end{aligned}
$$

with the $4 \times 4$ matrixes $\mathbf{A}^{\prime}, \mathbf{C}_{1, \pm 1}^{\prime}, \mathbf{C}_{2,0}^{\prime}$, and $\mathbf{C}_{2, \pm 2}^{\prime}$ being independent of $z$ and $\kappa_{H B M}$ but not of the half-period $\Omega$ and the handedness parameter $h$. Explicit representations of $\mathbf{M}^{\prime}(z, \omega)$ are straightforwardly derived but cumbersome; the reader is referred elsewhere for further details [33].

In the case of axial propagation (i.e., $\kappa_{H B M}=0$ ), the solution to Eq. (51) is conveniently expressed in closed form as [102]

$$
\mathbf{F}_{0}^{\prime}(z)=\exp \left(i \mathbf{A}^{\prime} z\right) \mathbf{F}_{0}^{\prime}(0)
$$

The nonaxial propagation case (i.e., $\kappa_{H B M} \neq 0$ ) is considerably more complicated to handle. However, Eq. (53) can be exploited to derive a solution to Eq. (51) in terms of a power series in $z$ [106]. As an alternative, a piecewise uniform approximation may also be implemented $[107,108]$.

The propensity of HBMs to exhibit characteristics related to negative refraction has been explored for the particular cases of chiral ferronematic materials (which are characterized by the constitutive relations in Eq. (44) with $\boldsymbol{\epsilon}_{H B M}$ and $\boldsymbol{\mu}_{H B M}$ having uniaxial forms, and the magnetoelectric dyadics $\xi_{H B M}$ and $\zeta_{H B M}$ being null valued) [27] and chiral ferrosmectic materials (which are characterized by the constitutive Eqs. (44) with $\boldsymbol{\epsilon}_{H B M}$ and $\boldsymbol{\mu}_{H B M}$ having biaxial forms, and the magnetoelectric dyadics $\xi_{H B M}$ and $\zeta_{H B M}$ being null valued) [28]. In both instances, it was observed that the structural handedness of the materials is effectively reversed when the real parts of the permittivity and permeability parameters are changed from positive to negative. That is, a structurally right/left-handed HBM with negative real permittivity and permeability parameters exhibits the circular Bragg phenomenon as though it were a structurally left/right-handed HBM with positive real permittivity and permeability parameters. Furthermore, when the real parts of the permittivity and permeability parameters are changed from positive to negative, the reflection and transmission coefficients undergo a phase reversal that can result 


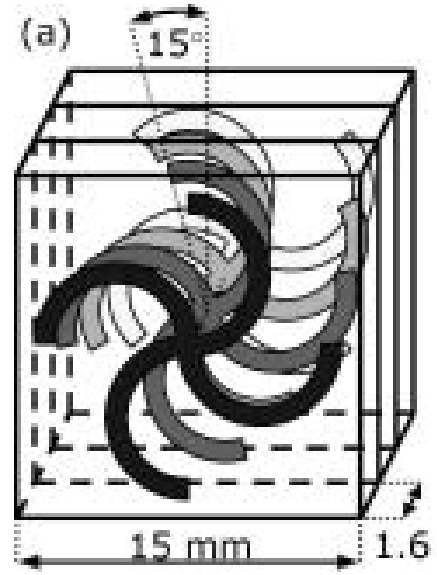

(b)

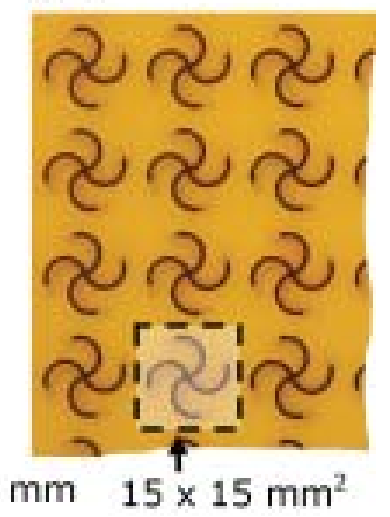

Fig. 12 The ambichiral metamaterial of Plum et al. [117]. The structure is based on layers of metallic rosettes. The rosettes in consecutive layers have a relative twist of 15 deg. (Reprinted with permission from Ref. 117. Copyright 2009 by the American Physical Society.)

in negative Goos-Hänchen shifts [28]. Such negative Goos-Hänchen shifts are a characteristic of materials that support NPV [110-113].

\subsection{Ambichirality}

Imagine a uniaxial crystalline sheet whose distinguished axis is oriented normal to the sheet's thickness direction. Now consider stack of such sheets with the angle between the distinguished axes of each pair of neighbouring sheets being $\pi / q, q=2,3, \ldots$ For $q>2$ this stack represents an ambichiral material, as originally conceived by Reusch [32] and later analyzed by Joly and Billard [114]. As is true for HBMs (which may be viewed as the limiting case $q \rightarrow \infty$ ), ambichiral materials can exhibit different Bragg resonances for different circular polarization states. ${ }^{\dagger}$ However, if the dielectric and magnetic dyadics of the underlying constituent materials are indefinite, the corresponding ambichiral material loses its ability to discriminate between different circular polarization states, provided that the number of constituent sheets is sufficiently large. This property — which arises because two of the four planewave components in each sheet are of the NPV type and two are of the positive-phase-velocity type - enables such an ambichiral material to function as a polarization-universal rejection filter [116].

The ambichiral architecture essentially underpins a recent report by Plum et al. [117] of a negatively refracting metamaterial, based on a stack of sheets each containing metallic rosettes with every sheet rotated about the normal axis with respect to the previous sheet in the stack by an angle less that $90 \mathrm{deg}$. An illustration is provided in Fig. 12. A similarly stacked chiral metamaterial that supports negative refraction, with a cross-wire microstructure, has been described by Zhou et al. [118].

\section{Closing Remarks}

The interaction of light with chiral metamaterials presents promising opportunities. In this review we have highlighted the propensity of these metamaterials to support negative refraction and closely related phenomenons. However, these opportunities come at a price: there are also formidable challenges, both experimental and theoretical, associated with chiral metamaterials. As we highlight in Sec. 2.5, the characterization of the complex behavior exhibited by these metamaterials requires careful treatment. In particular, optical properties cannot be characterized

\footnotetext{
${ }^{\dagger}$ The $q=2$ case - which corresponds to an equichiral material-is of little technological interest as such materials exhibit the same Bragg resonances for both circular polarization states [115].
} 

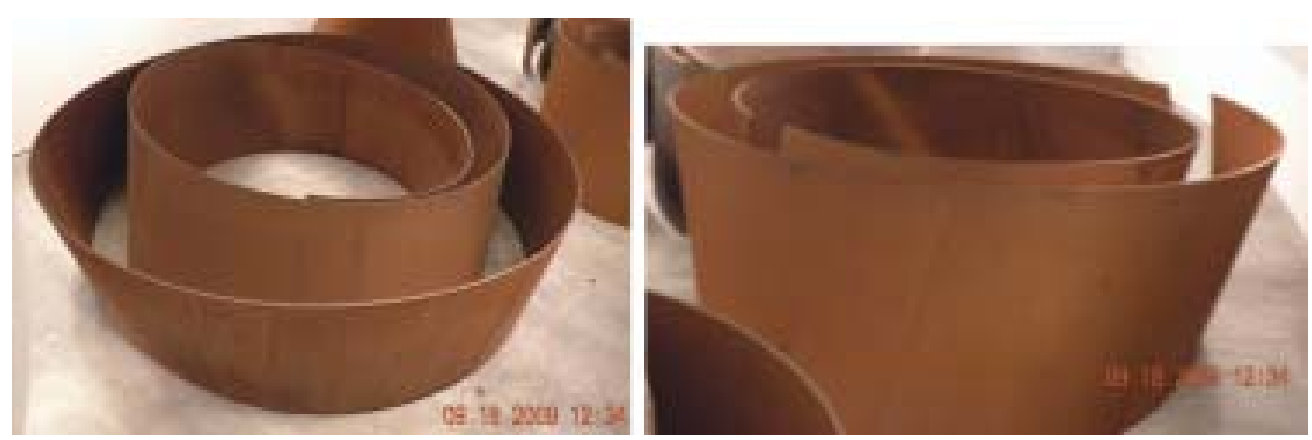

Fig. 13 Two of the eight sculptures from the permanent installation The Matter of Time by Richard Serra at the Guggenheim Museum Bilbao. The sculpture on the left may remind the reader of a Swiss roll, the one on the right of a double split-ring resonator, both structures commonplace in the literature on negatively refracting metamaterials. Were the metal sheets in both sculptures strictly vertical, both would be achiral.

by a simple scalar refractive index that refers only to one mode of propagation, along one direction only. Instead, multiple wavevectors, which may be directionally dependent, need to be considered. The comprehensive characterization of optical properties may involve significant distinctions still being made between counterposition, NPV, and negative refraction.

A perusal of ongoing experimental research on optical (frequency $\lesssim 500 \mathrm{THz}$ ) metamaterials shows that standard micro- and nano-fabrication techniques are being used to make them [12-15,100,119-122]. Metamaterials for operation at frequencies exceeding $500 \mathrm{THz}$ would have to be engineered on the nanoscale [15,119]. Remarkable optical effects can be expected from nanoengineered metamaterials, as nanotechnology for optics is a play of phase, length, and time [123]. As quantum-confinement effects will also emerge at higher frequencies [124], a strict scaling down of classical electromagnetics [125] may not suffice for designing both metamaterials and metamaterial-based devices. The associated difficulties are likely to be accentuated for asymmetric morphology at the nanoscale and, thus, for chiral metamaterials. To compensate, we must note that nanofabrication methods [126-128] are suited well to produce assemblies of different kinds of electrically small cells for multifunctional performance at the macroscopic scale.

While metamaterials (chiral or otherwise) have much to offer, after the past nine years or more of intensive research there still remains a wide gulf between the research frontier and practical applications [129]. One major issue to be tackled is the dissipation of electromagnetic energy in a material of reasonable thickness. Either pre-amplification or the incorporation of gain materials appears necessary, though we must note that the energy budget may then turn out to be economically impractical. Another major issue is the rapid variation of constitutive parameters with frequency in the frequency regimes of interest. This large dispersion could significantly distort pulse-modulated waves. Ingenuity and perseverance-perhaps, along with serendipity - are needed to find economically viable metamaterials that refract negatively.

\section{Acknowledgments}

We thank the authors of Refs. 54, 84, and 117 for providing us with figures from their papers. TGM thanks the Royal Academy of Engineering/Leverhulme Trust for partial financial support. AL thanks the Charles Godfrey Binder Endowment at Penn State for partial support of his research efforts.

We are happy to acknowledge parallels between ideas in the technoscientific and artistic worlds by drawing the reader's attention to Fig. 13 comprising photographs of two sculptures of Richard Serra installed at the Guggenheim Museum, Bilbao, Spain. 
Mackay and Lakhtakia: Negatively refracting chiral metamaterials ...

\section{References}

[1] J.-B. Biot and F. Arago, "Mémoire sur les affinités des corps pour la lumière et particulièrement sur les forces réfringentes des différents gaz," Mém. Inst. Fr. 7, 301-385 (1806).

[2] P. S. Neelakanta, Handbook of Electromagnetic Materials, CRC Press, Boca Raton, FL, USA (1995).

[3] A. Lakhtakia, Ed., Selected Papers on Linear Optical Composite Materials, SPIE Optical Engineering Press, Bellingham, WA, USA (1996).

[4] L. L. Beecroft and C. K. Ober, "Nanocomposite materials for optical applications," Chem. Mater. 9, 1302-1317 (1997).

[5] C. Brosseau, "Modelling and simulation of dielectrics heterostructures: a physical survey from an historical perspective," J. Phys. D: Appl. Phys. 39, 1277-1294 (2006).

[6] A. Lakhtakia and T. G. Mackay, "Meet the metamaterials," Optics \& Photonics News 18(1), 32-39 (2007).

[7] R. M. Walser, "Metamaterials: an introduction," in Introduction to Complex Mediums for Optics and Electromagnetics, W. S. Weiglhofer, and A. Lakhtakia, Eds., pp. 295-316, SPIE Press, Bellingham, WA, USA (2003).

[8] A. Lakhtakia, "Sculptured thin films as nanoengineered metamaterials," presented at Photonics 2008: International Conference on Fiber Optics and Photonics, New Delhi, India, December 14-17, 2008.

[9] D. R. Smith, W. J. Padilla, D. C. Vier, S. C. Nemat-Nasser, and S. Schultz, "Composite medium with simultaneously negative permeability and permittivity," Phys. Rev. Lett. 84, 4184-4187 (2000).

[10] R. A. Shelby, D. R. Smith, and S. Schultz, "Expermental verification of negative index of refraction," Science 292, 77-79 (2001).

[11] J. B. Pendry, "Negative refraction," Contemp. Phys. 45, 191-202 (2004).

[12] G. Dolling, M. Wegener, C. M. Soukoulis, and S. Linden, "Negative-index metamaterial at $780 \mathrm{~nm}$ wavelength," Opt. Lett. 32, 53-55 (2007).

[13] V. M. Shalaev, "Optical negative-index metamaterials," Nature Photon. 1, 41-48 (2007).

[14] J. Yao, Z. Liu, Y. Liu, Y. Wang, C. Sun, G. Bartal, A. M. Stacy, and X. Zhang, "Optical negative refraction in bulk metamaterials of nanowires," Science 321, 930 (2008).

[15] Y.-J. Jen, A. Lakhtakia, C.-W. Yu, and C.-T. Lin, "Vapor-deposited thin films with negative real refractive index in the visible regime," Opt. Exp. 17, 7784-7789 (2009).

[16] M. A. Noginov, "Compensation of surface plasmon loss by gain in dielectric medium," J. Nanophoton. 2, 021855 (2008).

[17] A. Bratkovsky, E. Ponizovskaya, S.-Y. Wang, P. Holmström, L. Thylén, Y. Fu, and H. Ågren, "A metal-wire/quantum-dot composite metamaterial with negative $\epsilon$ and compensated optical loss," Appl. Phys. Lett. 93, 193106 (2008).

[18] D. G. Stavenga, "Invertebrate superposition eyes - structures that behave like metamaterial with negative refractive index," J. Eur. Opt. Soc. Rapid Pub. 1, 06010 (2006).

[19] A. Pimenov, A. Loidl, K. Gehrke, V. Moshnyaga, and K. Samwer, "Negative refraction observed in a metallic ferromagnet in the gigahertz frequency range," Phys. Rev. Lett. 98, 197401 (2007).

[20] S. A. Ramakrishna, "Physics of negative refractive index materials," Rep. Prog. Phys. 68, 449-521 (2005).

[21] S. Tretyakov, I. Nefedov, A. Sihvola, S. Maslovski, and C. Simovski, "Waves and energy in chiral nihility," J. Electromag. Waves Applics. 17, 695-706 (2003).

[22] J. B. Pendry, "A chiral route to negative refraction," Science 306, 1353-1355 (2004).

[23] C. Monzon and D. W. Forester, "Negative refraction and focusing of circularly polarized waves in optically active media," Phys. Rev. Lett. 95, 123904 (2005). 
[24] T. G. Mackay, "Plane waves with negative phase velocity in isotropic chiral mediums," Microw. Opt. Technol. Lett. 45, 120-121 (2005). Corrections 47, 406 (2005).

[25] T. G. Mackay and A. Lakhtakia, "Plane wave with negative phase velocity in Faraday chiral mediums," Phys. Rev. E 69, 026602 (2004).

[26] T. G. Mackay and A. Lakhtakia, "Negative phase velocity in a material with simultaneous mirror-conjugated and racemic chirality characteristics," New J. Phys. 7, 65 (2005).

[27] A. Lakhtakia, "Reversal of circular Bragg phenomenon in ferrocholesteric materials with negative real permittivities and permeabilities," Adv. Mater. 14, 447-449 (2002).

[28] A. Lakhtakia, "Handedness reversal of circular Bragg phenomenon due to negative real permittivity and permeability," Opt. Exp. 11, 716-722 (2003).

[29] A. Lakhtakia, Beltrami Fields in Chiral Media, World Scientific, Singapore (1994).

[30] S. Chandrasekhar, Liquid Crystals, 2nd ed., Cambridge University Press, Cambridge, UK (1992).

[31] P. G. de Gennes and J. A. Prost, The Physics of Liquid Crystals, 2nd ed., Clarendon Press, Oxford, UK (1993).

[32] E. Reusch, "Untersuchung über Glimmercombinationen," Ann. Phys. Chem. Lpz. 138, 628-638 (1869).

[33] A. Lakhtakia and R. Messier, Sculptured Thin Films: Nanoengineered Morphology and Optics, SPIE Press, Bellingham, WA, USA (2005).

[34] J. C. Bose, "On the rotation of plane of polarisation of electric waves by a twisted structure," Proc. R. Soc. Lond. 63, 146-152 (1898). A facsimile reproduction is available in: O. N. Singh and A. Lakhtakia, Eds., Electromagnetic Fields in Unconventional Materials and Structures, Wiley, New York, NY, USA (2000).

[35] T. Ohno and S. Miyanishi, "Study of surface plasmon chirality induced by Archimedes' spiral grooves," Opt. Exp 14, 6285-6290 (2006).

[36] K. Jefimovs, N. Saito, Yu. Ino, T. Vallius, P. Vahimaa, J. Turunen, R. Shimano, M. Kauranen, Yu. Svirko, and M. Kuwata-Gonokami, "Optical activity in chiral gold nanogratings," Microelectron. Eng. 78-79, 448-451 (2005).

[37] S. Takahashi, A. Potts, D. Bagnall, N. I. Zheludev, and A. V. Zayats, "Near-field polarization conversion in planar chiral nanostructures," Opt. Commun. 255, 91-96 (2005).

[38] M. Decker, M. W. Klein, M. Wegener, and S. Linden, "Circular dichroism of planar chiral magnetic metamaterials," Opt. Lett. 32, 856-858 (2007).

[39] C. Zhang and T. J. Cui, "Negative reflections of electromagnetic waves in strong chiral medium," Appl. Phys. Lett. 91, 194101 (2007).

[40] M. Faryad and Q. A. Naqvi, "Cylindrical reflector in chiral medium supporting simultaneously positive phase velocity and negative phase velocity," J. Electromag. Waves Applics. 22, 563-572 (2008).

[41] T. G. Mackay and A. Lakhtakia, "Negative reflection in a Faraday chiral medium," Microw. Opt. Technol. Lett. 50, 1368-1371 (2008).

[42] T. G. Mackay and A. Lakhtakia, "Negative refraction, negative phase velocity, and counterposition in bianisotropic materials and metamaterials," Phys. Rev. B 79, 235121 (2009).

[43] M. W. McCall, A. Lakhtakia, and W. S. Weiglhofer, "The negative index of refraction demystified," Eur. J. Phys. 23, 353-359 (2002).

[44] R. A. Depine and A. Lakhtakia, "A new condition to identify isotropic dielectric-magnetic materials displaying negative phase velocity," Microw. Opt. Technol. Lett. 41, 315-316 (2004).

[45] A. Lakhtakia, V. V. Varadan, and V. K. Varadan, "A parametric study of microwave reflection characteristics of a planar achiral-chiral interface," IEEE Trans. Electromag. Compat. 28, 90-95 (1986).

[46] P. P. Banerjee and M. R. Chatterjee, "Negative index in the presence of chirality and material dispersion," J. Opt. Soc. Am. B 26, 194-202 (2009). 
[47] A. Baev, M. Samoc, P. N. Prasad, M. Krykunov, and J. Autschbach, "A quantum chemical approach to the design of chiral negative index materials," Opt. Exp. 15, 5730-5741 (2007).

[48] C. Zhang and T. J. Cui, "Chiral planar waveguide for guiding single-mode backward wave," Opt. Commun. 280, 359-363 (2007).

[49] C.-W. Qiu, N. Burokur, S. Zouhdi, and L.-W. Li, "Chiral nihility effects on energy flow in chiral materials," J. Opt. Soc. Am. A 25, 55-63 (2008).

[50] C. Sabah, "Left-handed chiral metamaterials," Central Eur. J. Phys. 6, 872-878 (2008).

[51] D.-H. Kwon, D. H. Werner, A. V. Kildishev, and V. M. Shalaev, "Material parameter retrieval procedure for general bi-isotropic metamaterials and its application to optical chiral negative-index metamaterial design," Opt. Exp. 16, 11822-11829 (2008).

[52] K. Matra and N. Wongkasem, "Left-handed chiral isotropic metamaterials: analysis and detailed numerical study," J. Opt. A: Pure Appl. Opt. 11, 074011 (2009).

[53] Q. A. Naqvi, "Fractional dual solutions to the Maxwell equations in chiral nihility medium," Opt. Commun. 282, 2016-2018 (2009).

[54] B. Wang, J. Zhou, T. Koschny, and C. M. Soukoulis, "Nonplanar chiral metamaterials with negative index," Appl. Phys. Lett. 94, 151112 (2009).

[55] T. G. Mackay and A. Lakhtakia, "Electromagnetic fields in linear bianisotropic mediums," Prog. Optics 51, 121-209 (2008).

[56] E. Charney, The Molecular Basis of Optical Activity, Krieger, Malabar, FL, USA (1985).

[57] A. Lakhtakia, Ed., Selected Papers on Natural Optical Activity, SPIE Optical Engineering Press, Bellingham, WA, USA (1990).

[58] R. Ro, V. V. Varadan, and V. K. Varadan, "Experimental study of chiral composites," Proc. SPIE 1558, 269-287 (1991).

[59] R. Luebbers, H. S. Langdon, F. Hunsberger, C. F. Bohren, and S. Yoshikawa, "Calculation and measurement of the effective chirality parameter of a composite chiral material over a wide frequency band," IEEE Trans. Antennas Propagat. 43, 123-130 (1995).

[60] Á. Gómez, A. Lakhtakia, J. Margineda, G. J. Molina-Cuberos, M. J. Núñez, J. A. Saiz Ipiña, A. Vegas, and M. A. Solano, "Full-wave hybrid technique for 3-D isotropicchiral-material discontinuities in rectangular waveguides: Theory and experiment," IEEE Trans. Microw. Theory Tech. 56, 2815-2825 (2008).

[61] W. Dong and L. Gao, "Negative refraction in chiral composite materials," J. Appl. Phys. 104, 023537 (2008).

[62] T. G. Mackay and A. Lakhtakia, "Simultaneous negative- and positive-phase-velocity propagation in an isotropic chiral medium," Microw. Opt. Technol. Lett. 49, 1245-1246 (2007).

[63] A. Lakhtakia and T. G. Mackay, "Infinite phase velocity as the boundary between positive and negative phase velocities," Microw. Opt. Technol. Lett. 41, 165-166 (2004).

[64] C. Zhang and T. J. Cui, "Spatial dispersion and energy in a strong chiral medium," Opt. Exp. 15, 5114-5119 (2007).

[65] A. Alù, F. Bilotti, N. Engheta, and L. Vegni, "Theory and simulations of a conformal omnidirectional subwavelength metamaterial leaky-wave antenna," IEEE Trans. Antennas Propagat. 55, 1698-1708 (2007).

[66] M. G. Silveirinha and N. Engheta, "Transporting an image through a subwavelength hole," Phys. Rev. Lett. 102, 103902 (2009).

[67] R. Liu, Q. Cheng, T. Hand, J. J. Mock, T. J. Cui, S. A. Cummer, and D. R. Smith, "Expermental demonstration of electromagnetic tunneling through an epsilonnear-zero metamaterial at microwave frequencies," Phys. Rev. Lett. 100, 023903 (2008).

[68] F. Bilotti, S. Tricarico, and L. Vegni, "Electromagnetic cloaking devices for TE and TM polarizations," New J. Phys. 10, 115035 (2008).

[69] A. Lakhtakia, "An electromagnetic trinity from 'negative permittivity' and 'negative permeability'," Int. J. Infrared Millimeter Waves 23, 813-818 (2002). 
[70] H. C. Chen, Theory of Electromagnetic Waves, McGraw-Hill, New York, NY, USA (1983).

[71] B. Lax and K. J. Button, Microwave Ferrites and Ferrimagnetics, McGraw-Hill, New York, NY, USA (1962).

[72] N. Engheta, D. L. Jaggard, and M. W. Kowarz, "Electromagnetic waves in Faraday chiral media," IEEE Trans. Antennas Propagat. 40, 367-374 (1992).

[73] W. S. Weiglhofer and A. Lakhtakia, "The correct constitutive relations of chiroplasmas and chiroferrites," Microw. Opt. Technol. Lett. 17, 405-408 (1998).

[74] W. S. Weiglhofer and T. G. Mackay, "Numerical studies of the constitutive parameters of a chiroplasma composite medium," Arch. Elektr. Übertrag. 54, 259-265 (2000).

[75] T. G. Mackay, A. Lakhtakia, and W. S. Weiglhofer, "Ellipsoidal topology, orientation diversity and correlation length in bianisotropic composite mediums," Arch. Elektr. Übertrag. 55, 243-251 (2001).

[76] L. Tsang and J. A. Kong, "Scattering of electromagnetic waves from random media with strong permittivity fluctuations," Radio Sci. 16, 303-320 (1981).

[77] T. G. Mackay, A. Lakhtakia, and W. S. Weiglhofer, "Strong-property-fluctuation theory for homogenization of bianisotropic composites: formulation," Phys. Rev. E 62, 60526064 (2000). Corrections: 63, 049901 (2001).

[78] B. Michel and A. Lakhtakia, "Strong-property-fluctuation theory for homogenizing chiral particulate composites," Phys. Rev. E 51, 5701-5707 (1995).

[79] H. C. van de Hulst, Light Scattering by Small Particles, Dover, New York, NY, USA (1981).

[80] T. G. Mackay, "Lewin's homogenization formula revisited for nanocomposite materials," J. Nanophoton. 2, 029503 (2008).

[81] M. C. K. Wiltshire, J. B. Pendry, and J. V. Hajnal, "Chiral Swiss rolls show a negative refractive index," J. Phys.: Condens. Matter 21, 292201 (2009).

[82] I. V. Semchenko, S. A. Khakhomov, and S. A. Tretyakov, "Chiral metamaterial with unit negative refraction index," Eur. Phys. J. Appl. Phys. 46, 32607 (2009).

[83] X. Cai, R. Zhu, and G. Hu, "Experimental study for metamaterials based on dielectric resonators and wire frame," Metamaterials 2, 220-226 (2008).

[84] S. Zhang, Y.-S. Park, J. Li, X. Lu, W. Zhang, and X. Zhang, "Negative refractive index in chiral metamaterials," Phys. Rev. Lett. 102, 023901 (2009).

[85] A. Lakhtakia and W. S. Weiglhofer, "Electromagnetic waves in a material with simultaneous mirror-conjugated and racemic chirality characteristics," Electromagnetics 20, 481-488 (2000). (The first negative sign on the right side of Eq. (26) of this paper should be replaced by a positive sign).

[86] A. H. Cooke, S. J. Swithenby, and M. R. Wells, "Magnetoelectric measurements on holmium phosphate, $\mathrm{HoPO}_{4}$," Int. J. Magnetism 4, 309-312 (1973).

[87] G. Gorodetsky, R. M. Hornreich, and B. M. Wanklyn, "Statistical mechanics and critical behavior of the magnetoelectric effect in $\mathrm{GdVO}_{4}$," Phys. Rev. B 8, 2263-2267 (1973).

[88] C. M. Krowne, "Electromagnetic theorems for complex anisotropic media," IEEE Trans. Antennas Propagat. 32, 1224-1230 (1984).

[89] P. A. Belov, "Backward waves and negative refraction in uniaxial dielectrics with negative dielectric permittivity along the anisotropy axis," Microw. Opt. Technol. Lett. 37, 259263 (2003).

[90] T. G. Mackay and A. Lakhtakia, "Negative refraction, negative phase velocity and counterposition in bianisotropic materials and metamaterials," Phys. Rev. B 79, 235121 (2009).

[91] Y. Zhang, B. Fluegel, and A. Mascarenhas, "Total negative refraction in real crystals for ballistic electrons and light," Phys. Rev. Lett. 91, 157404 (2003).

[92] A. Lakhtakia and M. W. McCall, "Counterposed phase velocity and energy-transport velocity vectors in a dielectric-magnetic uniaxial medium," Optik 115, 28-30 (2004).

[93] A. Serdyukov, I. Semchenko, S. Tretyakov, and A. Sihvola, Electromagnetics of Bi-anisotropic Materials, Gordon and Breach, Amsterdam, The Netherlands (2001). 
[94] M. M. I. Saadoun and N. Engheta, "A reciprocal phase shifter using novel pseudochiral or omega medium," Microw. Opt. Technol. Lett. 5, 184-188 (1992).

[95] T. M. Grzegorczyk and J. A. Kong, "Electrodynamics of moving media inducing positive and negative refraction," Phys. Rev. B 74, 033102 (2006).

[96] A. J. Hoffman, L. Alekseyev, S. S. Howard, K. J. Franz, D. Wasserman, V. A. Podolskiy, E. E. Narimanov, D. L. Sivco, and C. Gmachl, "Negative refraction in semiconductor metamaterials," Nature Mater. 6, 946-950 (2007).

[97] J. Yao, Z. Liu, Y. Liu, Y. Wang, C. Sun, G. Bartal, A. M. Stacy, and X. Zhang, "Optical negative refraction in bulk metamaterials of nanowires," Science 321, 930 (2008).

[98] X. Chen, B.-I. Wu, J. A. Kong, and T. M. Grzegorczyk, "Retrieval of the effective constitutive parameters of bianisotropic metamaterials," Phys. Rev. E 71, 046610 (2005).

[99] M. S. Rill, C. Plet, M. Thiel, I. Staude, G. von Freymann, S. Linden, and M. Wegener, "Photonic metamaterials by direct laser writing and silver chemical vapour deposition," Nature Mater. 7, 543-546 (2008).

[100] M. S. Rill, C. E. Kriegler, M. Thiel, G. von Freymann, S. Linden, and M. Wegener, "Negative-index bianisotropic photonic metamaterial fabricated by direct laser writing and silver shadow evaporation," Opt. Lett. 34, 19-21 (2009).

[101] S. T. Chui, W. Wang, L. Zhou, and Z. F. Lin, "Longitudinal elliptically polarized electromagnetic waves in off-diagonal magnetoelectric split-ring composites," J. Phys.: Condens. Matter 21, 292202 (2009).

[102] A. Lakhtakia and W. S. Weiglhofer, "On light propagation in helicoidal bianisotropic mediums," Proc. R. Soc. Lond. A 448, 419-437 (1995). Corrections: 454, 3275 (1998).

[103] S. Pursel, M. W. Horn, M. C. Demirel, and A. Lakhtakia, "Growth of sculptured polymer submicronwire assemblies by vapor deposition," Polymer 46, 9544-9548 (2005).

[104] J. A. Sherwin and A. Lakhtakia, "Nominal model for the optical response of a chiral sculptured thin film infiltrated with an isotropic chiral fluid," Opt. Commun. 214, 231245 (2002).

[105] J. A. Sherwin and A. Lakhtakia, "Nominal model for the optical response of a chiral sculptured thin film infiltrated by an isotropic chiral fluid-oblique incidence," Opt. Commun. 222, 305-329 (2003).

[106] A. Lakhtakia and W. S. Weiglhofer, "Further results on light propagation in helicoidal bianisotropic mediums: oblique propagation," Proc. R. Soc. Lond. A 453, $93-105$ (1997). Corrections: 454, 3275 (1998).

[107] M. Schubert and C. M. Herzinger, "Ellipsometry on anisotropic materials: Bragg conditions and phonons in dielectric helical thin films," Phys. Status Solidi, A 188, 1563-1575 (2001).

[108] J. A. Polo, Jr. and A. Lakhtakia, "Comparison of two methods for oblique propagation in helicoidal bianisotropic mediums," Opt. Commun. 230, 369-386 (2004).

[109] C. W. Oseen, "The theory of liquid crystals," J. Chem. Soc. Faraday Trans. II 29, 883899 (1933).

[110] A. Lakhtakia, "On planewave remittances and Goos-Hänchen shifts of planar slabs with negative real permittivity and permeability," Electromagnetics 23, 71-75 (2003).

[111] P. R. Berman, "Goos-Hänchen shift in negatively refractive media," Phys. Rev. E 66, 067603 (2002). Corrections: 71, 039903 (2005).

[112] J. A. Kong, B.-I. Wu, and Y. Zhang, "Lateral displacement of a Gaussian beam reflected from a grounded slab with negative permittivity and permeability," Appl. Phys. Lett. 80, 2084-2086 (2002).

[113] A. Lakhtakia, "Positive and negative Goos-Hänchen shifts and negative phase-velocity mediums (alias left-handed materials)," Arch. Elektr. Übertrag. 58, 229-231 (2004).

[114] G. Joly and J. Billard, "Quelques champs électromagnétiques dans les piles de Reusch. II. Piles éclairées sous l'incidence normale par des ondes monochromatiques planes et uniformes," J. Opt. (Paris) 13, 227-238 (1982). 
[115] I. J. Hodgkinson, A. Lakhtakia, Q. h. Wu, L. De Silva, and M. W. McCall, "Ambichiral, equichiral and finely chiral layered structures," Opt. Commun. 239, 353-358 (2004).

[116] A. Lakhtakia, "Polarization-universal rejection filtering by ambichiral structures made of indefinite dielectric-magnetic materials," Phys. Scr. 77, 055401 (2008).

[117] E. Plum, J. Zhou, J. Dong, V. A. Fedotov, T. Koschny, C. M. Soukoulis, and N. I. Zheludev, "Metamaterial with negative index due to chirality," Phys. Rev. B 79, 035407 (2009).

[118] J. Zhou, J. Dong, B. Wang, T. Koschny, M. Kafesaki, and C. M. Soukoulis, "Negative refractive index due to chirality," Phys. Rev. B 79, 121104(R) (2009).

[119] R. Abdeddaïm, G. Guida, A. Priou, B. Gallas, and J. Rivory, "Negative permittivity and permeability of gold square nanospirals," Appl. Phys. Lett. 94, 081907 (2007).

[120] A. V. Kildishev, W. Cai, U. K. Chettiar, H.-K. Yuan, A. K. Sarychev, V. P. Drachev, and V. M. Shalaev, "Negative refractive index in optics of metal-dielectric composites," J. Opt. Soc. Am. B 23, 423-433 (2006).

[121] C. Helgert, C. Menzel, C. Rockstuhl, E. Pshenay-Severin, E.-B. Kley, A. Chipouline, A. Tünnermann, F. Lederer, and T. Pertsch, "Polarization-independent negative-index metamaterial in the near infrared," Opt. Lett. 34, 704-706 (2009).

[122] U. Huebner, J. Petschulat, E. Pshenay-Severin, A. Chipouline, T. Pertsch, C. Rockstuhl, and F. Lederer, "Negative-index materials: Two approaches for nanofabricated metamaterials," Microelectron. Eng. 86, 1138-1141 (2009).

[123] A. Lakhtakia and J. B. Geddes III, "Nanotechnology for optics is a phase-length-time sandwich," Opt. Eng. 43, 2410-2417 (2004).

[124] J. M. Martinez-Duart, R. J. Martín-Palma, and F. Agulló-Rueda, Nanotechnology for Microelectronics and Optoelectronics, Elsevier, Amsterdam, The Netherlands (2006).

[125] A. Lakhtakia, "Scaling of fields, sources, and constitutive properties in bianisotropic media," Microw. Opt. Technol. Lett. 7, 328-330 (1994).

[126] S. Y. Chou, P. R. Krauss, and P. J. Renstrom, "Imprint lithography with 25-nanometer resolution," Science 272, 85-87 (1996).

[127] M. W. Horn, M. D. Pickett, R. Messier, and A. Lakhtakia, "Blending of nanoscale and microscale in uniform large-area sculptured thin-film architectures," Nanotechnol. 15, 303-310 (2004).

[128] D. Van Gough, A. T. Juhl, and P. V. Braun, "Programming structure into 3D nanomaterials," Mater. Today 12(6), 28-35 (2009).

[129] P. Ikonen, "Electrically small metamaterial-based antennas-have we seen any real practical benefits?," presented at EuCAP 2009 3rd European Conference on Antennas and Propagation, Berlin, Germany, March 23-27, 2009. http://arXiv.org/0902.1820.

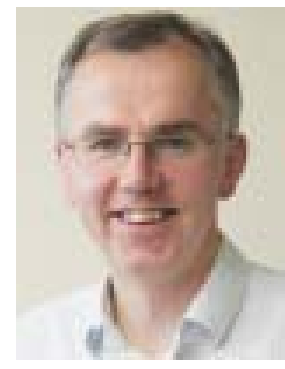

Tom G. Mackay completed his $\mathrm{PhD}$ studies in applied mathematics under the supervision of Werner S. Weiglhofer in the Department of Mathematics, University of Glasgow, in 2001. Then he moved to the Department of Mathematics and Statistics (now called the School of Mathematics), University of Edinburgh, where he is currently a Reader. He is also an adjunct professor in the Department of Engineering Science and Mechanics, Pennsylvania State University. At present his research interests include homogenization, complex materials, metamaterials, and sculptured thin films. He has received financial support from The Carnegie Trust for The Universities of Scotland, The Nuffield Foundation, and EPSRC (UK); he held a Royal Society of Edinburgh/Scottish Executive Support Research Fellowship in 2006/2007, and he holds a Royal Academy of Engineering/Leverhulme Trust Senior Research Fellowship for 2009/2010. For further details see http://www.maths.ed.ac.uk/ tgm. 


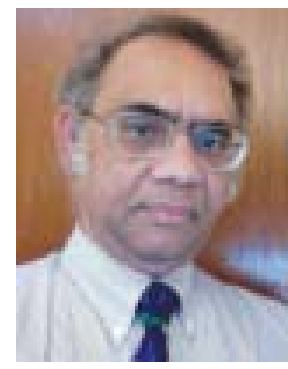

Akhlesh Lakhtakia obtained a bachelor of technology degree in electronics engineering from the Banaras Hindu University, Varanasi, India, in 1979, and master of science and doctor of philosophy degrees in electrical engineering from the University of Utah, Salt Lake City, in 1981 and 1983, respectively. Thereafter, he joined the faculty of the Pennsylvania State University, where he became distinguished professor of engineering science and mechanics in January 2004. From 2004 to 2007 he also held the rank of a visiting professor of physics at Imperial College, London. In 2006, he became the Charles Godfrey Binder (Endowed) Professor of Engineering Science and Mechanics. He has published more than 650 journal articles; contributed 18 chapters to research books and encyclopedias; edited, coedited, authored, or coauthored 13 books and 10 conference proceedings; reviewed for 111 journals; serves on the editorial boards of four electromagnetics journals; was the Editor-in-Chief of the international journal Speculations in Science and Technology from 1993 to 1995; and became the first Editor-in-Chief of the online Journal of Nanophotonics launched by SPIE in 2007. He served as an international lecturer for the International Commission for Optics and the Optical Society of America was twice a visiting professor of physics at Universidad de Buenos Aires, a visiting professor of physics at the University of Otago, and a visiting fellow in mathematics at the University of Glasgow. He headed the IEEE EMC Technical Committee on Nonsinusoidal Fields from 1992 to 1994 and is a fellow of the Optical Society of America, SPIE, and the Institute of Physics (UK). He also served as the 1995 Scottish Amicable Visiting Lecturer at the University of Glasgow. He received the PSES Outstanding Research Award in 1996, the PSES Premier Research Award in 2008, and the PSES Outstanding Advising Award in 2005. For his research on sculptured thin films and complex-medium electromagnetics, he received the Faculty Scholar Medal in Engineering in 2005 at Penn State, and a Doctor of Science degree in Electronics Engineering from the Banaras Hindu University in 2006. Nanotech Briefs recognized him in 2006 with a Nano 50 Award for Innovation. The University of Utah made him a Distinguished Alumnus in 2007. His current research interests lie in the electromagnetics of complex materials, sculptured thin films, chiral nanotubes, nanoengineered metamaterials, biomimetics, and negative refraction. At Penn State, he codeveloped a course on green engineering for undergraduate engineering students, as well as a course on fundamentals of engineering principles and design for preservice elementary schoolteachers. 